\title{
Bifurcation and Nonlinear Analysis of a Time-delayed Thermoacoustic System
}

DOI:

10.1016/j.cnsns.2016.08.006

\section{Document Version}

Accepted author manuscript

Link to publication record in Manchester Research Explorer

\section{Citation for published version (APA):}

Yanga, X., Turan, A., \& Shenghui, L. (2017). Bifurcation and Nonlinear Analysis of a Time-delayed

Thermoacoustic System. Communications in Nonlinear Science and Numerical Simulation, 44, 229-244.

https://doi.org/10.1016/j.cnsns.2016.08.006

\section{Published in:}

Communications in Nonlinear Science and Numerical Simulation

\section{Citing this paper}

Please note that where the full-text provided on Manchester Research Explorer is the Author Accepted Manuscript or Proof version this may differ from the final Published version. If citing, it is advised that you check and use the publisher's definitive version.

\section{General rights}

Copyright and moral rights for the publications made accessible in the Research Explorer are retained by the authors and/or other copyright owners and it is a condition of accessing publications that users recognise and abide by the legal requirements associated with these rights.

\section{Takedown policy}

If you believe that this document breaches copyright please refer to the University of Manchester's Takedown Procedures [http://man.ac.uk/04Y6Bo] or contact uml.scholarlycommunications@manchester.ac.uk providing relevant details, so we can investigate your claim.

\section{OPEN ACCESS}




\title{
Bifurcation and Nonlinear Analysis of a Time-delayed Thermoacoustic System
}

\author{
Xiaochuan Yang $^{a^{*}}$, Ali Turan ${ }^{\mathrm{a}}$, Shenghui Lei ${ }^{\mathrm{b}}$ \\ ${ }^{\mathrm{a}}$ School of Mechanical, Aerospace and Civil Engineering, \\ The University of Manchester, P.O. Box 88, Manchester M60 1QD, UK \\ ${ }^{\mathrm{b}}$ Thermal Management Research Group, Efficient Energy Transfer ( $\left.\eta E T\right)$, \\ Bell Labs, Alcatel-Lucent, Dublin 15, Ireland
}

\begin{abstract}
In this paper, of primary concern is a time-delayed thermoacoustic system, viz. a horizontal Rijke tube. A continuation approach is employed to capture the nonlinear behavior inherent to the system. Unlike the conventional approach by the Galerkin method, a dynamic system is naturally built up by discretizing the acoustic momentum and energy equations incorporating appropriate boundary conditions using a finite difference method. In addition, the interaction of Rijke tube velocity with oscillatory heat release is modeled using a modified form of King's law. A comparison of the numerical results with experimental data and the calculations reported reveals that the current approach can yield very good predictions. Moreover, subcritical Hopf bifurcations and fold bifurcations are captured with the evolution of dimensionless heat release coefficient, generic damping coefficient and time delay. Linear stability boundary, nonlinear stability boundary, bistable region and limit cycles are thus determined to gain an understanding of the intrinsic nonlinear behaviors.
\end{abstract}

Keywords: Rijke tube; time delay; thermoacoustic instability; bifurcation analysis

\footnotetext{
* Corresponding author

Tel:(+44)-1612754368 Email address: xiaochuan.yang@postgrad.manchester.ac.uk ( X. Yang)
} 


\section{Introduction}

Thermoacoustic instability widely exists in various combustion systems, such as propulsion systems, rocket motors, industrial burners, gas turbine engines etc. [1-3]. It arises from the interaction between the heat release and acoustic pressure or velocity oscillations within the combustion system. Under proper conditions, the acoustic perturbations would be strengthened and large amplitude limit cycles could occur. The energy density is usually so high that the resulting limit cycles would cause severe damages to the combustors. Hence, the oscillations have to be either avoided or controlled to an acceptable level. This requires a through and deep understanding of the instability mechanism, including the triggering, damping, flame-acoustic coupling etc. There has been a long history on the research of thermoacoustic instability. Following the very early observations of Sondhauss [4] and Rijke [5], Rayleigh defined the condition to trigger this instability, known as the Rayleigh criteria [6]. Thenceforth, this topic had long attracted the attention of researchers, especially with the development of rockets, jet engines and other industrial combustion systems. The pioneering theoretical work done by Cuclik [7-8] analyzed the nonlinear behavior of acoustic waves within a combustion chamber and provided a formal framework to study the growth and limiting amplitude of acoustic waves. Dowling made tremendous contribution on the understanding of acoustic-combustion interaction [9-10], control of thermoacoustic instability [11] and application in aeronautic and power generation combustors [12]. Morgans and her co-workers [1314] used a network model combined with flame describing function to predict the nonlinear thermoacoustic behaviour in combustors. Juniper [15] employed adjoint looping of the nonlinear governing equations as well as an optimization routine to study the triggering mechanism, including the non-normality, transient growth and 
bypass transition. Heckl [16] developed an empirical model for the nonlinear behavior of both heat release and the reflection coefficients and this model was widely used to predict the limit cycle. Hoejmakers, Doey and Nijmeijer [17-19] utilized binary classification theory and network models to predict the stability of a thermoacoustic system. The intrinsic flame stability and flame-acoustic coupling were also investigated in real burners. Polifke and co-workers have also done much work on the instability study of thermoacoustic systems using Hybrid CFD/ low-order modelling [20], state-space models [21], adjoint Helmholtz solver [22], and frequency domain system model [23]. Poinsot and colleagues conducted large eddy simulation and experiments on the measurement of flame transfer function [24-25], evaluation of dynamic flame response [26-27] and acoustic analysis of thermoacoustic instability [28-29] in gas turbine combustion chambers. Candel [30-34] also explored the interactions between acoustics and swirling flames and came up with a unified framework for nonlinear combustion instability analysis. Sujith and his co-workers implemented analytical and experimental study and also bifurcation analysis to understand the dynamic behaviours involved in thermoacoustic systems, such as nonnormality [35], nonlinearity [36], route to chaos [37], intermittency [38-39] etc. Yang has done numerous fundamental, numerical and experimental work on thermoacoustic instability encountered in gas turbine [40], liquid rocket engines [41] and liquidfuelled propulsion systems [42]. Campa and Camporeale [43-45] used Finite Element Method to predict the acoustically driven combustion instabilities and investigated the influence of flame and burner transfer matrix on the instability mode and frequencies. More work in this field can be found in review papers [46-49].

Since the instability is generally not desirable, it has to be controlled or avoided. There have been many passive or active control methods, such as Helmholtz resonator 
[50-51], perforated liner [52], jet injection [53], fuel injection [54] and acoustic feedback [3]. However, these methods are still not adequate enough to be applied into systems like jet engines because of either inefficiency or the lack of suitable actuators for operation in such harsh working environments. Thus, an alternative approach is to avoid the thermoacoustic oscillations by defining a safe operation region. Within this region, the oscillations would either not occur or retain at sufficiently low amplitudes. Due to the strong nonlinear characteristics, the system behaviours are generally functions of operation parameters. Industrial combustors are often experimentally tested under different working conditions to find the safe operation region, which is extremely expensive and complicated. Therefore, it would be a good choice to computationally estimate the safe operation region, which thus raises the demand to come up with accurate numerical methods. Since practical combustors normally involve complex geometries, turbulent flow and combustion, it would be a good starting point to investigate the numerical method in a simple thermoacoustic system. Rijke tube has been a classical tool to study the thermoacoustic instability. It usually consists of an open-end tube and heat source inside it. When the heat source is placed in certain positions along the tube, sound would emit from the tube. The sound is generated due to the transfer from unsteady heat release to acoustic energy. Despite the simplicity in structure, it contains rich nonlinear behaviors, such as bifurcation, limit cycle, quasiperiodicity and chaos, which make it an excellent example for the study of thermoacoustic instability $[11,55-56]$.

In the past decades, Rijke tube has been extensively studied to understand the intrinsic nonlinear behavior of the thermoacoustic instability. Hantschk and Vortmeyer [57] investigated self-excited thermoacoustic instabilities in the Rijke tube using a commercial CFD code. Two different kinds of Rijke tubes were modelled and 
the results showed good agreement with experiments. Non-linearity in the heat flux from the heating source to the flow was found to determine the limit cycle amplitudes. Matveev [58-59] combined linear theory and thermal analysis to predict the linear stability boundaries in a horizontal Rijke tube. A special form of the nonlinear heat transfer function was introduced to extend the method to nonlinear stability analysis. Hysteresis phenomenon was reported in the stability boundary and limit cycles were predicted as observed in experiments. Ananthkrishnan et al. [60] obtained the reduced-order models to capture the global behavior of chamber dynamics via truncating the modal expansions and determined the number of modes required for accurate results. Heckl and Howe [61] conducted stability analysis of the Rijke tube by making use of a Green's function. Oscillations were described in terms of the eigenmodes of an integral equation derived using the Green's function and the predictions of stability behavior were in line with Rayleigh's criterion. Balasubramanian and Sujith [35] studied the role of non-normality and nonlinearity in thermoacoustic system in a Rijke tube using the heat release model from Heckl [16]. It was shown that the non-normality inherent in the thermoacoutic system could result in transient growth of oscillations which can trigger nonlinearities in the system. Subramanian et al. [62] conducted bifurcation analysis of the dynamic behaviors of a horizontal Rijke tube and obtained bifurcation plots as a function of different system parameters. No/linear stability boundaries and other nonlinear phenomena were also observed in the analysis of the thermoacoustic system. Noble et al. [63] described a data-driven nonlinear and chaos theory-based analysis of thermoacoustic instabilities in a simple Rijke tube. It only relied on experimental data with no implicit assumptions. PLIFH measurement of $\mathrm{OH}$ radical at the rate of $2500 \mathrm{~Hz}$ was used to 
capture the thermoacoustic instability modes appeared in the Rijke tube. Chaotic behavior was identified in the thermoacoustic instability.

In order to study thermoacoustic instability, most models adopt the conservation equations for mass, momentum and energy to represent the nature of thermoacoustic system. These equations are in the form of partial differential equations (PDEs) and need to be discretized and solved using proper numerical methods. Galerkin method [64] has been the most commonly employed technique in previous research. Nevertheless, in present study, a novel approach named Method of Lines (MOL) [65] was employed to convert the governing PDEs into a series of to ordinary differential equations (ODEs). It has seldom been used before in this field owing to the complexity of discretization and expensive computational cost. As shown in later sections, good predictions can be gained in comparison with the conventional method. Most importantly, this approach could precisely capture the intrinsic nonlinear behaviours compared with measurements as well as other predictions.

In this paper, a horizontal Rijke tube with heat source is employed for stability study. MOL is adopted for to discretize the governing equations. A linear multistep method (LMS-method) and Newton iteration method [66-67] are used to calculate the characteristic roots of the dynamic system for linear stability study. Numerical continuation method is utilized to obtain bifurcation diagrams for investigating nonlinear behavior including the Hopf bifurcation, fold bifurcation and limit cycles. With a limited increase of computational cost, the approach paves a different way to investigate the dynamic behaviours and in/stability of thermoacoustic system. It could also be generalized for more complex combustion and thermoacoustic systems. Therefore, it, as a useful tool, can guide the design and optimization of practical combustion systems. 


\section{Physical Model and Governing Equations}

\subsection{Physical Model}

In practice, the Rijke tube is often oriented vertically, in which a base flow is driven by natural convection. For the purpose of neglecting this complicated convection, in this paper, a primary concern is a horizontal Rijke tube to study the instability of thermoacoustic system.

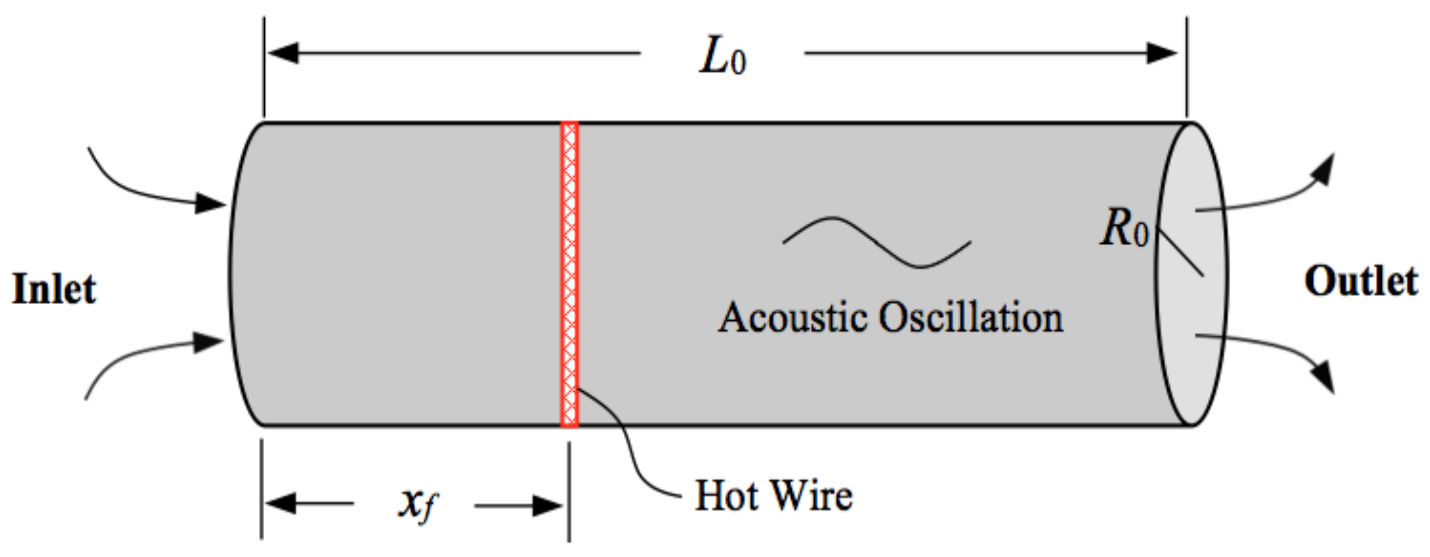

Fig. 1 Schematic of a classical horizontal Rijke tube

Figure 1 shows a schematic of a horizontal Rijke tube, which is an open-end cylinder with $L$ in length and $R_{0}$ in radium. A base flow driven by an external fan passes through the tube and is heated up by a hot wire gauze being placed at the position of $x_{f}$ from the inlet. Naturally, the tube displays an infinite number of acoustic modes. It was discovered by Rayleigh [68] that the thermal energy could be transferred to acoustic energy as long as they are in phase and the acoustic oscillations can be further strengthened. However, Matveev [58] argued that these oscillations could be damped due to energy dissipation through acoustic boundary layers at the wall, acoustic radiation from the open ends and convection of sound by the mean flow. Hence, it can be expected that the resulting nonlinearity can make this timedelayed system display considerable nonlinear behavior. 


\subsection{Governing Equations}

In this study, it is assumed that the fluid is a perfect, inviscid and non-heatconducting gas and therefore the influence of the mean flow and mean temperature gradients can be ruled out and the acoustic damping primarily results from the acoustic boundary layer and acoustic radiation mentioned in Section 2.1. Thus, the one-dimensional governing equations for the acoustic momentum and energy are

$$
\begin{gathered}
\rho_{0} \frac{\partial \tilde{u}}{\partial \tilde{t}}+\frac{\partial \tilde{p}}{\partial \tilde{x}}=0 \\
\frac{\partial \tilde{p}}{\partial \tilde{t}}+\gamma p_{0} \frac{\partial \tilde{u}}{\partial \tilde{x}}+\xi \frac{c_{0}}{L_{0}} \tilde{p}=(\gamma-1) \tilde{\dot{Q}}
\end{gathered}
$$

where $x$ and $t$ are streamwise location and time respectively; $\rho, u$ and $p$ are the fluid density, velocity and pressure respectively; the subscript 0 represents the unperturbed variables and the tilde $\sim$ denotes the perturbed variables; $\gamma, \xi, c_{0}$ and $L_{0}$ are the heat capacity ratio, generic acoustic damping coefficient, speed of sound and length of the tube respectively; $\tilde{\dot{Q}}$ is the rate of heat release perturbation per unit volume. This heat release can modeled using a modified form of King's law [16] given as

$$
\tilde{\dot{Q}}=\frac{2 L_{w}\left(T_{w}-T_{0}\right)}{S \sqrt{3}}\left(\pi \lambda c_{v} \rho_{0} \frac{\mathrm{d}_{w}}{2}\right)^{\frac{1}{2}}\left(\left|\frac{u_{0}}{3}+\tilde{u}_{f}(\tilde{t}-\tilde{\tau})\right|^{\frac{1}{2}}-\left(\frac{u_{0}}{3}\right)^{\frac{1}{2}}\right) \tilde{\delta}_{D}\left(\tilde{x}-\tilde{x}_{f}\right)
$$

where $L_{w}, d_{w}$ and $T_{w}$ represent the length, diameter and temperature of the hot wire gauze, respectively; $\lambda$ and $c_{v}$ are the fluid thermal conductivity and heat capacity at constant volume respectively; $T_{0}$ is the temperature of the unperturbed base flow; $S$ is the cross-sectional area of the tube; $\tilde{\tau}$ is the time delay; $\tilde{\delta}_{D}\left(\tilde{x}-\tilde{x}_{f}\right)$ is the Dirac Delta function to narrow the heat-release region specifically at the wire position $x_{f}$. The generic damping coefficient [69] is defined as 


$$
\xi=\pi\left(\frac{S}{L_{0}^{2}}+\frac{L}{R_{0}} \sqrt{\frac{2}{\pi}} \frac{\sqrt{v}+\sqrt{\chi}(\gamma-1)}{\sqrt{c_{0} L_{0}}}\right)
$$

where $v$ is the kinematic viscosity and $\chi$ is the thermal diffusivity.

The nondimensional variables are defined as,

$$
u=\frac{\tilde{u}}{u_{0}} ; p=\frac{\tilde{p}}{p_{0} \gamma M} ; x=\frac{\tilde{x}}{L_{0}} ; t=\frac{\tilde{t}}{L_{0} / c_{0}} ; \delta_{D}\left(x-x_{f}\right)=\tilde{\delta}_{D}\left(\tilde{x}-\tilde{x}_{f}\right) L_{0}
$$

where $p_{0}=\rho_{0} R_{\mathrm{u}} T_{0}$ for the ideal gas, $R_{\mathrm{u}}$ is the universal gas constant, $c_{0}=\left[\gamma R_{\mathrm{u}} T_{0}\right]^{1 / 2}$ and $M$ is the Mach number $\left(=u_{0} / c_{0}\right)$. With these definitions, one can substitute and obtain the governing equations Eqns. (1) and (2) in nondimensional form as

$$
\begin{gathered}
\frac{\partial u}{\partial t}+\frac{\partial p}{\partial x}=0 \\
\frac{\partial p}{\partial t}+\frac{\partial u}{\partial x}+\xi p-\beta \delta_{D}\left(x-x_{f}\right)\left(\left|\frac{1}{3}+u_{f}(t-\tau)\right|^{\mid \frac{1}{2}}-\left|\frac{1}{3}\right|^{\frac{1}{2}}\right)=0
\end{gathered}
$$

where $\beta$ is the heat release coefficient defined as

$$
\beta=\frac{1}{p_{0} \sqrt{u_{0}}} \frac{(\gamma-1)}{\gamma} \frac{2 L_{w}\left(T_{w}-T_{0}\right)}{S \sqrt{3}}\left(\pi \lambda c_{v} \rho_{0} \frac{\mathrm{d}_{w}}{2}\right)^{\frac{1}{2}}
$$

Clearly, this coefficient as a combination covers all the details of the fluid, the hotwire gauze and the tube.

In the study, the Rijke tube is open at both ends and therefore the boundary conditions at inlet (denoted as $x=0$ ) and outlet (denoted as $x=1$ ) become

$$
\begin{gathered}
\left.\frac{\partial u}{\partial x}\right|_{x=0,1}=0 \\
\left.p\right|_{x=0,1}=0
\end{gathered}
$$

It should be pointed that these boundary conditions enable the problem of interest to be a well-posed initial value problem within time domain. 
The acoustic energy per unit volume, $E$, as used by Juniper [15] can be expressed as in a nondimensional form

$$
E=\frac{1}{2} u^{2}+\frac{1}{2} p^{2}
$$

\section{Numerical Approach}

\subsection{Discretization Method}

To study dynamic behavior of the time-delayed system of Rijke tubes, a dynamic system constituting several ordinary differential equations (ODEs) should be constructed from the partial differential equations Eqns. (6) and (7). In the past, the Galerkin method was extensively employed, for which two sets of presumed basis functions for $u$ and $p$ were used to convert Eqns. (6) and (7) to ODEs for each acoustic mode. Thus, the selections of basis sets and mode number are expected to have significant influence on the results. In this paper, the approach originally employed is the Method of Lines (MOL) to build up a dynamic system for the Rijke tube. The basic idea of the MOL is to directly discretize the spatial variable $x$ in PDEs, but keep the temporal variable $t$ be continuous. It should be pointed out that the resulting complexity of discretization and expensive computation cost are still affordable considering the availability of high computational resources nowadays.

Besides, apart from the approximation of $\partial u / \partial x$ and $\partial p / \partial x$ along axis direction, it also involves in Galerkin method to approximate the nonlinear term $\left|\frac{1}{3}+u_{f}(t-\tau)\right|^{\frac{1}{2}}-\left|\frac{1}{3}\right|^{\frac{1}{2}}$ and the following term $u_{f}(t-\tau)[15,72]$, which might introduce more errors. However, in MOL approach, this term is very easy and straightforward to be implemented in the equation and requires no further approximation. Since it is the only nonlinear term in the dynamic system, its implementation is of crucial importance for the prediction of the inherent nonlinear characteristics. 
To obtain the unsteady solution structure, the governing equations Eqns. (6) and (7) were discretized by a finite difference method. The $x$ domain along the length of the tube is divided into $N$ points indexed as $x_{i}(i=1,2, \ldots, N)$. Thus there were $(N-1)$ parts with an identical interval of $\Delta x=1 /(N-1)$. Second-order central difference scheme was used to discretize the spatial derivatives $\partial u / \partial x$ and $\partial p / \partial x$.

For the internal points $x_{i}(i=2, \ldots, N-1)$, a series of ODE was obtained

$$
\begin{aligned}
& \dot{u}(i, t)=-\frac{p(i+1, t)-p(i-1, t)}{2 \Delta x} \\
& \dot{p}(i, t)=\left\{\begin{array}{l}
-\frac{u(i+1, t)-u(i-1, t)}{2 \Delta x}-\xi p(i, t), i \neq x_{f} \\
-\frac{u(i+1, t)-u(i-1, t)}{2 \Delta x}-\xi p(i, t)+\beta\left(\left|\frac{1}{3}+u(i, t-\tau)\right|^{\frac{1}{2}}-\left|\frac{1}{3}\right|^{\frac{1}{2}}\right), i=x_{f}
\end{array}\right.
\end{aligned}
$$

Particularly, a second-order upwind difference scheme was employed to deal with the Neumann boundary conditions for $u$ at $x_{1}$ and $x_{N}$, whereas specific values were given for the Dirichlet boundary conditions of $p$.

$$
\begin{aligned}
& \left.\frac{\partial u}{\partial x}\right|_{x=0}=\frac{-3 u(1, t)+4 u(2, t)-u(3, t)}{2 \Delta x}=0 \\
& \left.\frac{\partial u}{\partial x}\right|_{x=N}=\frac{3 u(N, t)-4 u(N-1, t)+u(N-2, t)}{2 \Delta x}=0
\end{aligned}
$$

Therefore, $u$ and $p$ at boundaries could be written as

$$
\begin{aligned}
& u(1, t)=\frac{4 u(2, t)-u(3, t)}{3} \\
& u(N, t)=\frac{4 u(N-1, t)-u(N-2, t)}{3} \\
& p(1, t)=p(N, t)=0
\end{aligned}
$$

By substituting these four variables into Eqns. (12) and (13), the ODE system would be closed and all the variables $u$ and $p$ could be solved. Within this dynamic system, there are $2 N$ variables and four bifurcation parameters, i.e. $\beta, \xi, x_{f}, \tau$. 


\subsection{Bifurcation Analysis Method}

To carry out the bifurcation analysis of the time-delayed thermoacoustic system, a numerical continuation method [66-67] was employed.

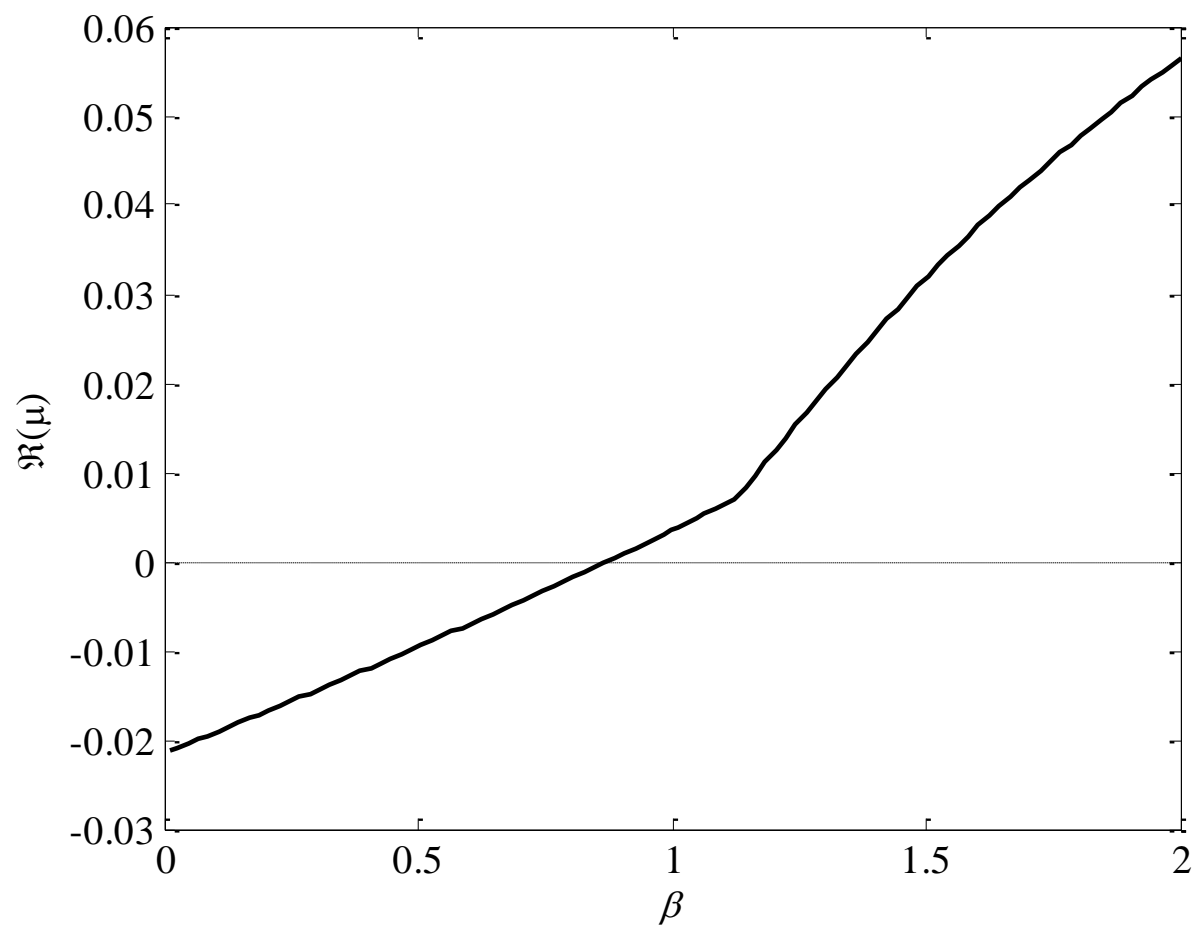

Fig. 2 Real parts of the rightmost characteristic roots versus $\beta$ for the system $\left(\zeta=0.043, x_{f}=0.3, \tau=0.02\right)$

First, the system was linearized around the pre-determined steady state (equilibrium) solution and the corresponding eigenvalues of the linearized system, namely the roots of the characteristic equation were calculated. These roots were first approximated by a linear multistep method (LMS-method) and then corrected using a Newton iteration method [67]. Mathematically, the rightmost characteristic root, or the characteristic root with the maximal real part, conclusively indicates whether or not the system is stable. If the rightmost characteristic root crosses zero as a bifurcation parameter marches on (as shown in Fig. 2), bifurcation may arise. Specifically, if a pair of complex conjugate roots crosses the imaginary axis, a Hopf bifurcation point appears as shown in Fig. 3(a). If a real root marches across zero, a fold bifurcation point presents as shown in Fig. 3(b). 


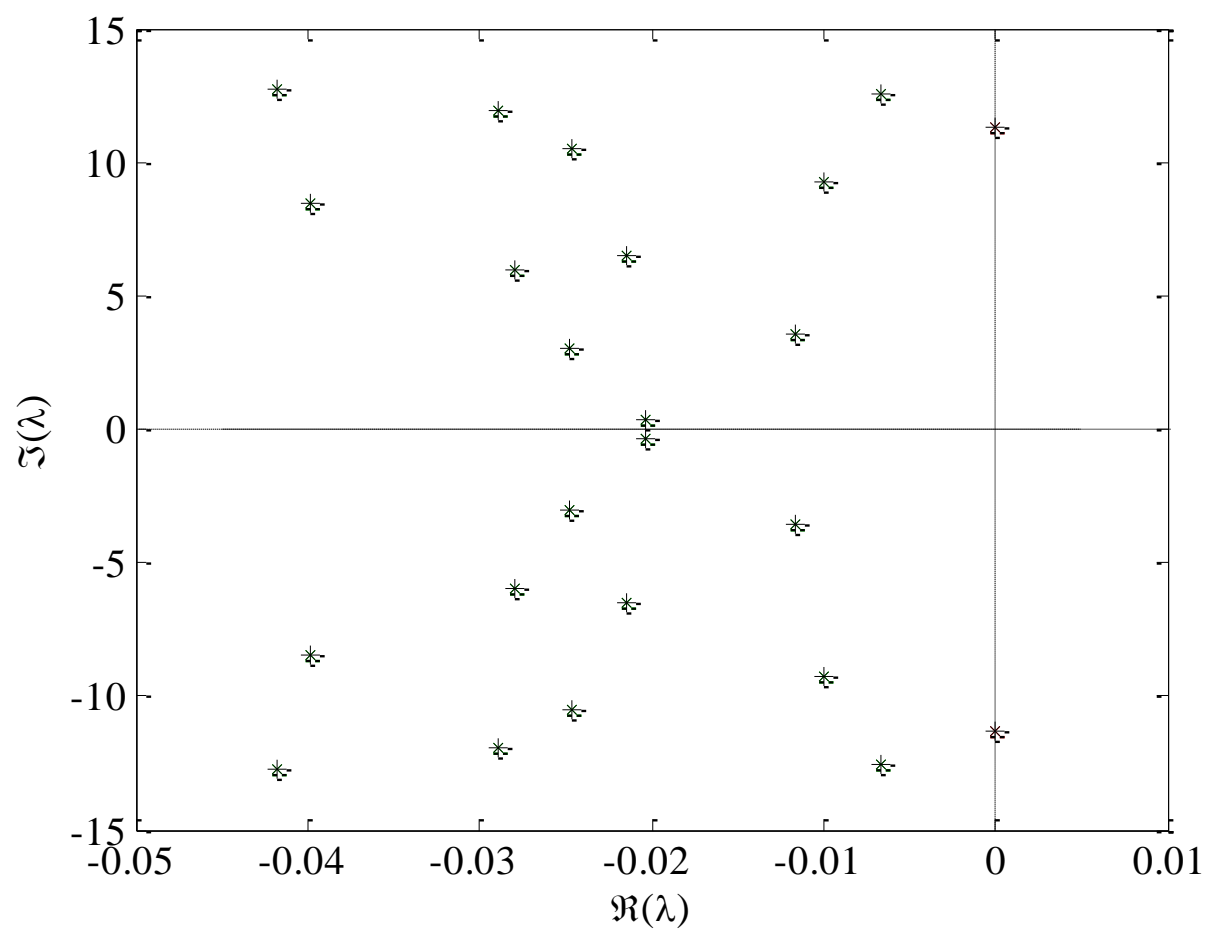

(a)

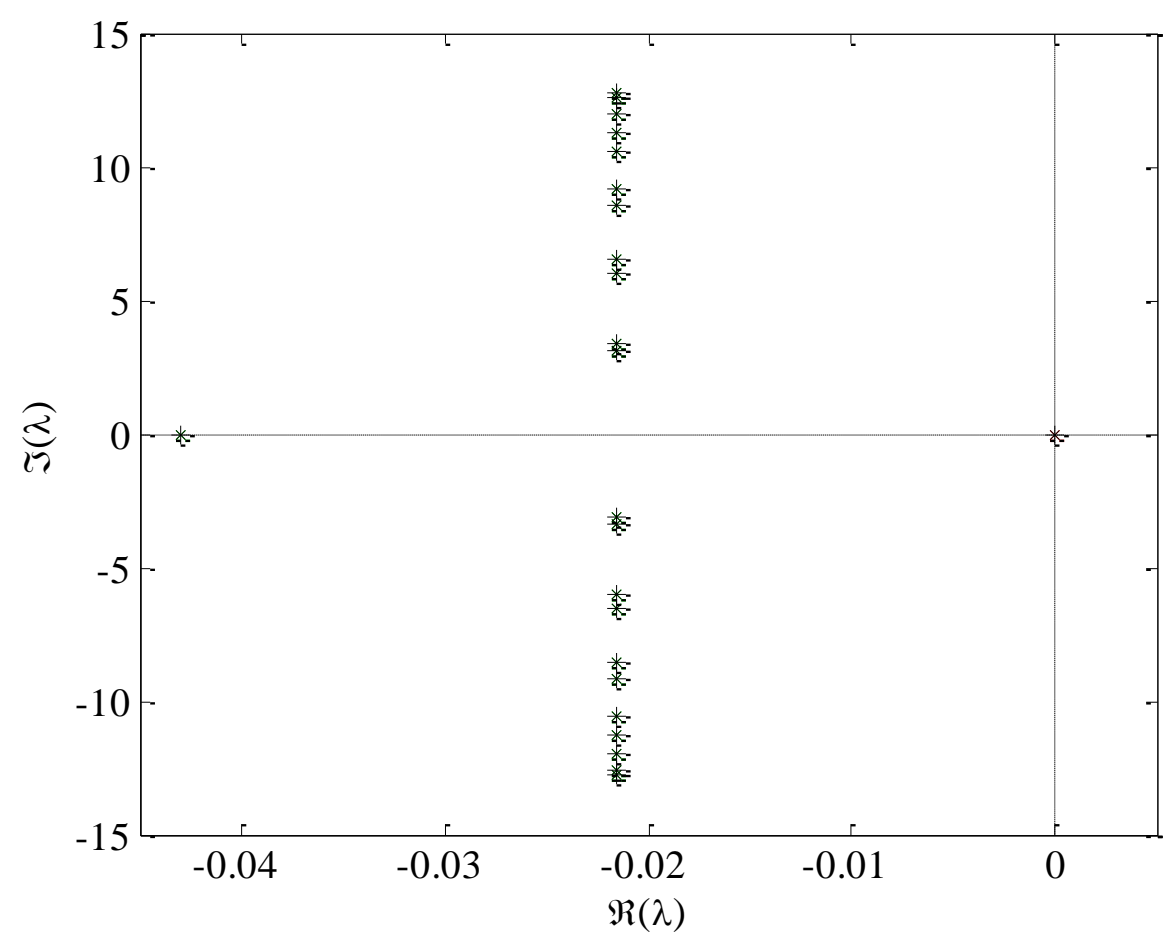

(b)

Fig. 3 (a) Characteristic roots at a Hopf point $\left(\beta_{H}=0.874\right)$ and (b) Characteristic roots at a fold point $\left(\beta_{f}\right.$ $=0.815)\left(\zeta=0.043, x_{f}=0.3, \tau=0.02\right)$ 
Furthermore, a prediction-correction approach [70-71] was employed to capture all the branches starting from the bifurcation point. Moreover, the Floquet-multiplier scheme was utilized to determine the stability of each branch, for which the solution is stable only if the moduli of all the multipliers are less than unity, as demonstrated in Fig. 4.

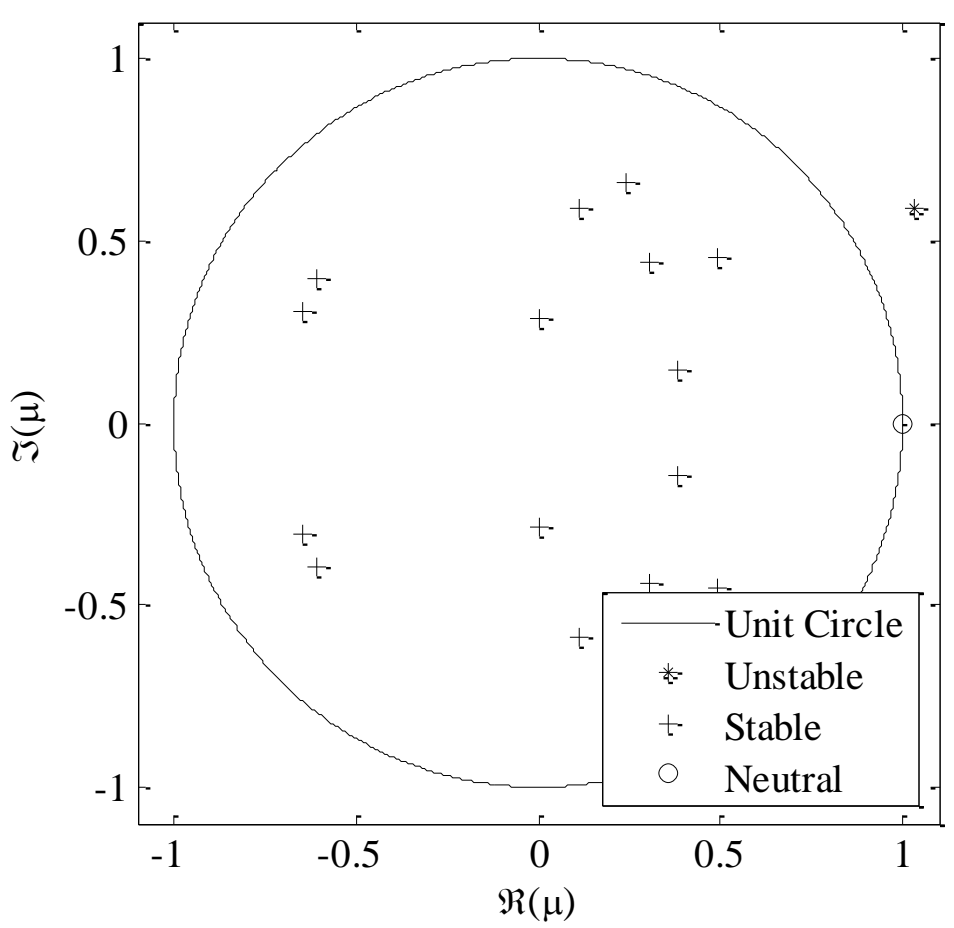

Fig. 4 Floquet Multipliers for an unstable periodic solution

\subsection{Grid Independence Check}

In Section 3.1, the governing equations were discretized into a series of ODEs using the finite difference method. The resolution of discretization will have influence on the convergence and accuracy of the solutions. Hence, the grid independence needs to be checked primarily to assure accuracy. As shown in Fig. 5, there is little change for the bifurcation diagram when the number of discretization points increase to $N=120$ and beyond. To compromise between the accuracy and speed, the $x$ domain is thus discretized into 120 points for the following bifurcation analysis. 


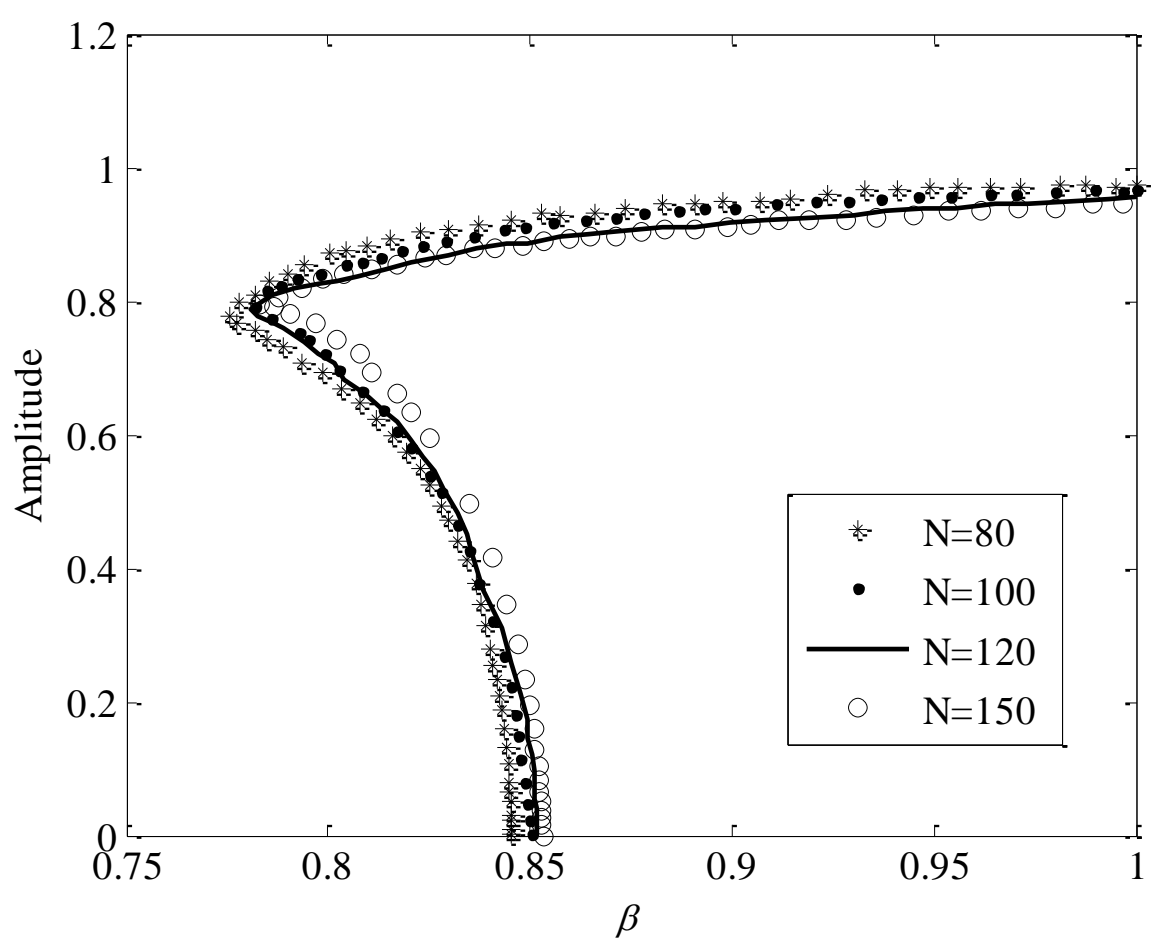

Fig. 5 Bifurcation diagrams as a function of $\beta$ using different discretization points

$$
\left(\zeta=0.043, x_{f}=0.4, \tau=0.02\right)
$$

\section{Results and Discussion}

In this section, the nonlinear behaviors of the Rijke tube thermoacoustic system are analyzed in detail. Different bifurcation diagrams are obtained to explore the effect of the system parameters including the heat release coefficient $\beta$, the generic damping coefficient $\zeta$ and the time delay $\tau$.

\subsection{Effect of Heat Release}

The heat release coefficient $\beta$ is a dimensionless parameter comprising all the details of the base flow, the hot wire and the tube. The effect of varying the heat release coefficient on the dynamic behavior of the system is presented by the bifurcation diagram shown in Fig. 6. Using different measurement for the limit cycles, they display the same tendency and the critical points are the same in both figures. 
Figure 6 presents a subcritical Hopf bifurcation and is similar to the bifurcation diagrams reported in $[33,62,72]$. The stability of each periodic solution was determined by Floquet multipliers, as discussed in Section 3.2. The steady state solution is stable for $\beta<0.874$. At the Hopf bifurcation point $\beta_{H}\left(\beta_{H}=0.874\right)$, the system loses stability and small amplitude periodic solutions (limit cycle) emerge from it. These limit cycles are unstable and will be stabilized through a 'turning point' or referred to as a fold bifurcation point $\left(\beta_{f}=0.815\right)$. When $\beta<\beta_{f}$, the steady state solution is stable for perturbations of any magnitude. In the region of $\beta_{f}<\beta<\beta_{H}$, linearly stable steady state solution, small-amplitude unstable periodic solutions and large-amplitude stable limit cycles coexist to form a bistable region, which is susceptible to triggering.

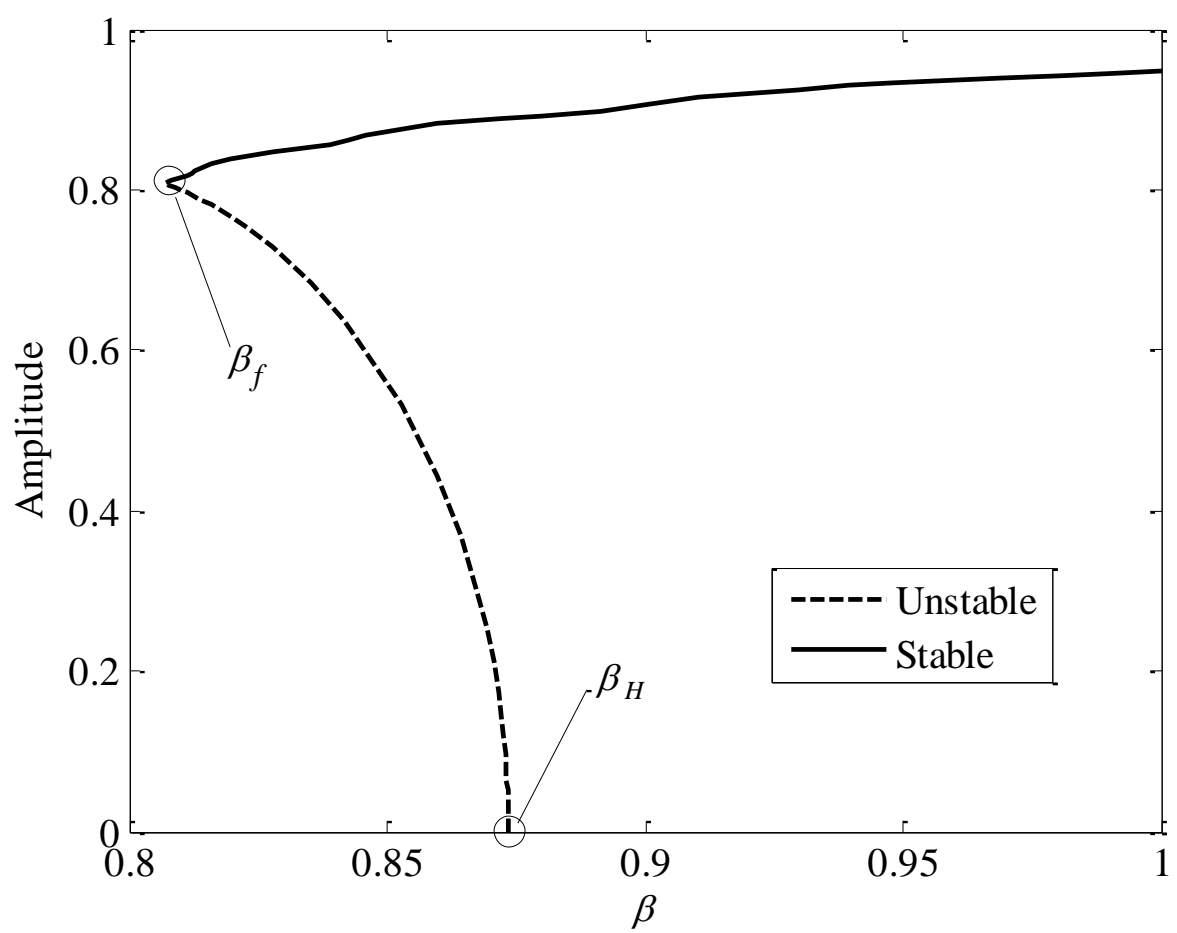

(a) 


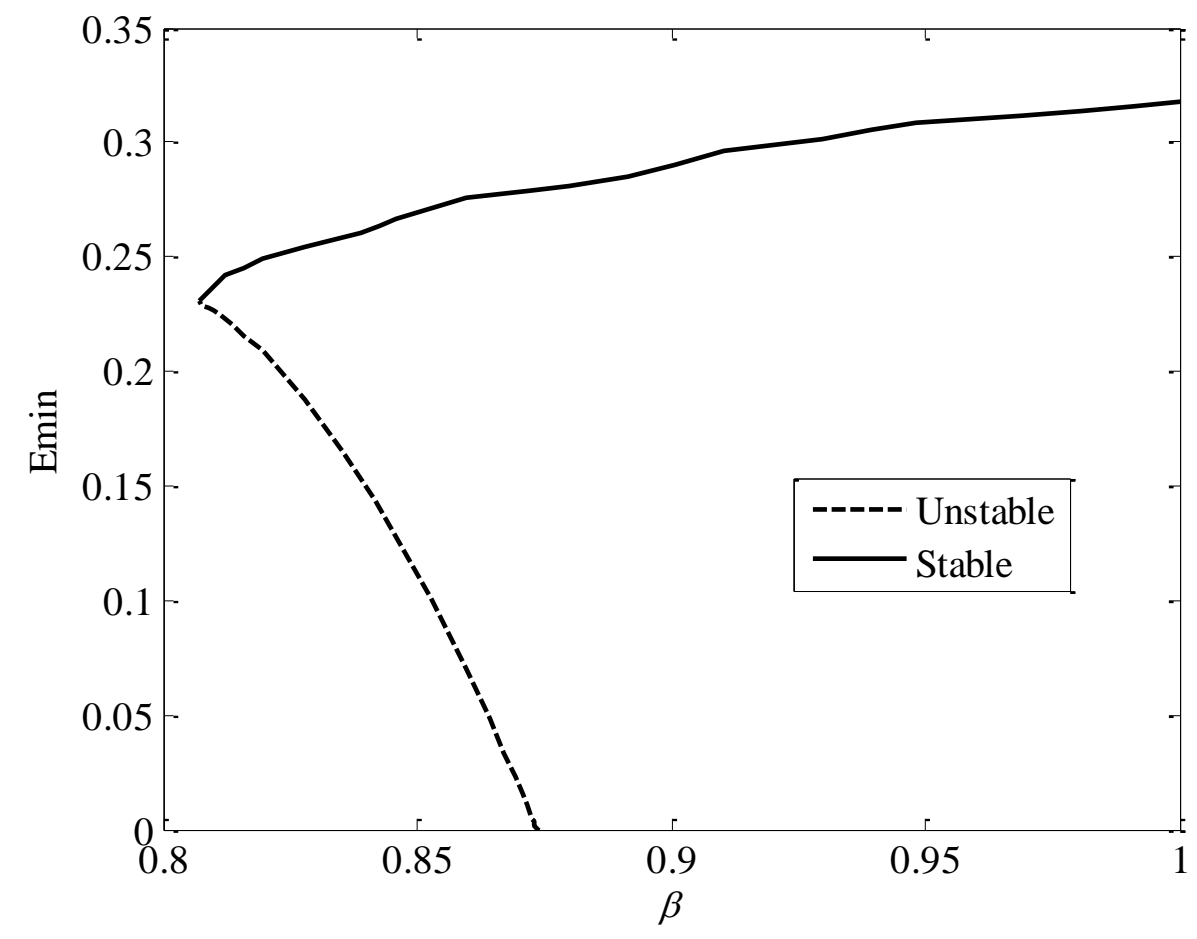

(b)

Fig. 6 Bifurcation diagrams as a function of $\beta\left(\zeta=0.043, x_{f}=0.3, \tau=0.02\right)$. (a) the peak-to-peak amplitude of velocity mode versus $\beta$, where dashed line indicates the unstable periodic solution, solid line indicates the stable periodic solution; (b) the dimensionless minimum acoustic energy on periodic solutions versus $\beta$.

In the Rijke tube, the increase of $\beta$ can be achieved by increasing the electrical supply of the hot wire gauze. The resulting strengthened heat release perturbation could act as a driving mechanism for the thermoacoustic instability. For a small value of $\beta$, the heat release perturbation can be damped by the system and the steady state solution is stable. With the growth of $\beta$, Hopf bifurcation occurs and it leads to the oscillating flow in the flow. A small perturbation may become a self-sustained largeamplitude oscillation.

\subsection{Effect of Time Delay}

The bifurcation diagram for a varying time delay is shown in Fig. 7. Similar to Fig. 6, the bifurcation is also a subcritical Hopf bifurcation. A small time delay below $\tau_{f}$ is 
always stable, while a large time delay over $\tau_{H}$ is always unstable. Between $\tau_{f}$ and $\tau_{H}$ is the bistable region. Since a time delay is the time taken for the change of acoustic velocity to get reflected in heat release perturbation[72], a decreased time delay would lead to a faster heat transfer between the heat source and air flow. Consequently, the air would quickly attain the same temperature as the heat source, which eventually reduces and eliminates the heat transfer and diminishes the acoustic oscillation. It can be concluded that decreased time delay has a stabilizing effect on the system. The time delay here was estimated by Lighthill [73]

$$
\tilde{\tau}=0.2 \frac{\tilde{d}_{w}}{\tilde{u}_{0}}
$$

Thus, under presently employed uniform configuration, a small time delay corresponds to a large speed of the mean flow. To obtain a small time delay in Rijke tube, the speed of base flow should be enhanced properly.

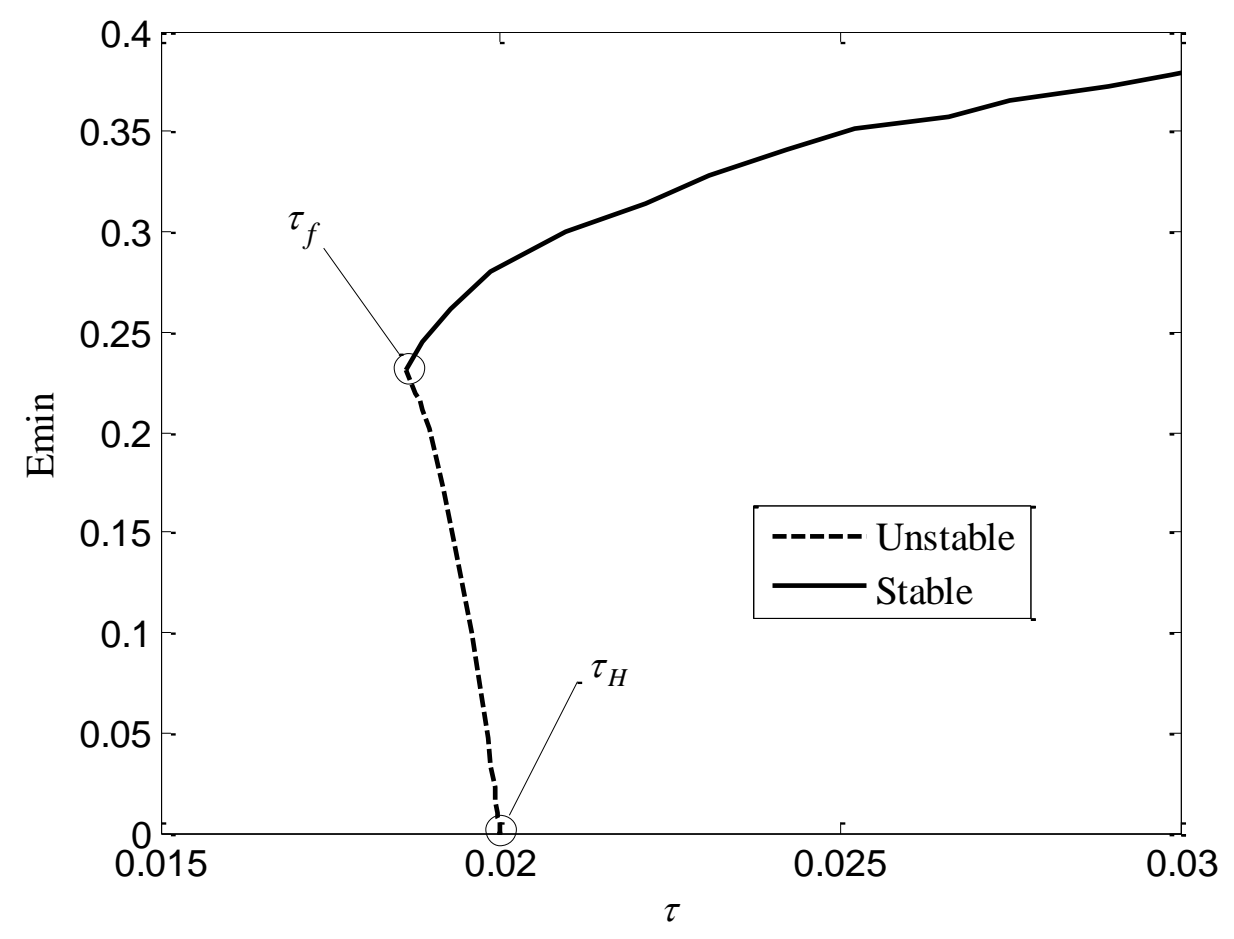

Fig. 7 Bifurcation diagrams as a function of $\tau\left(\zeta=0.043, x_{f}=0.3, \beta=0.874\right)$ 


\subsection{Effect of Damping}

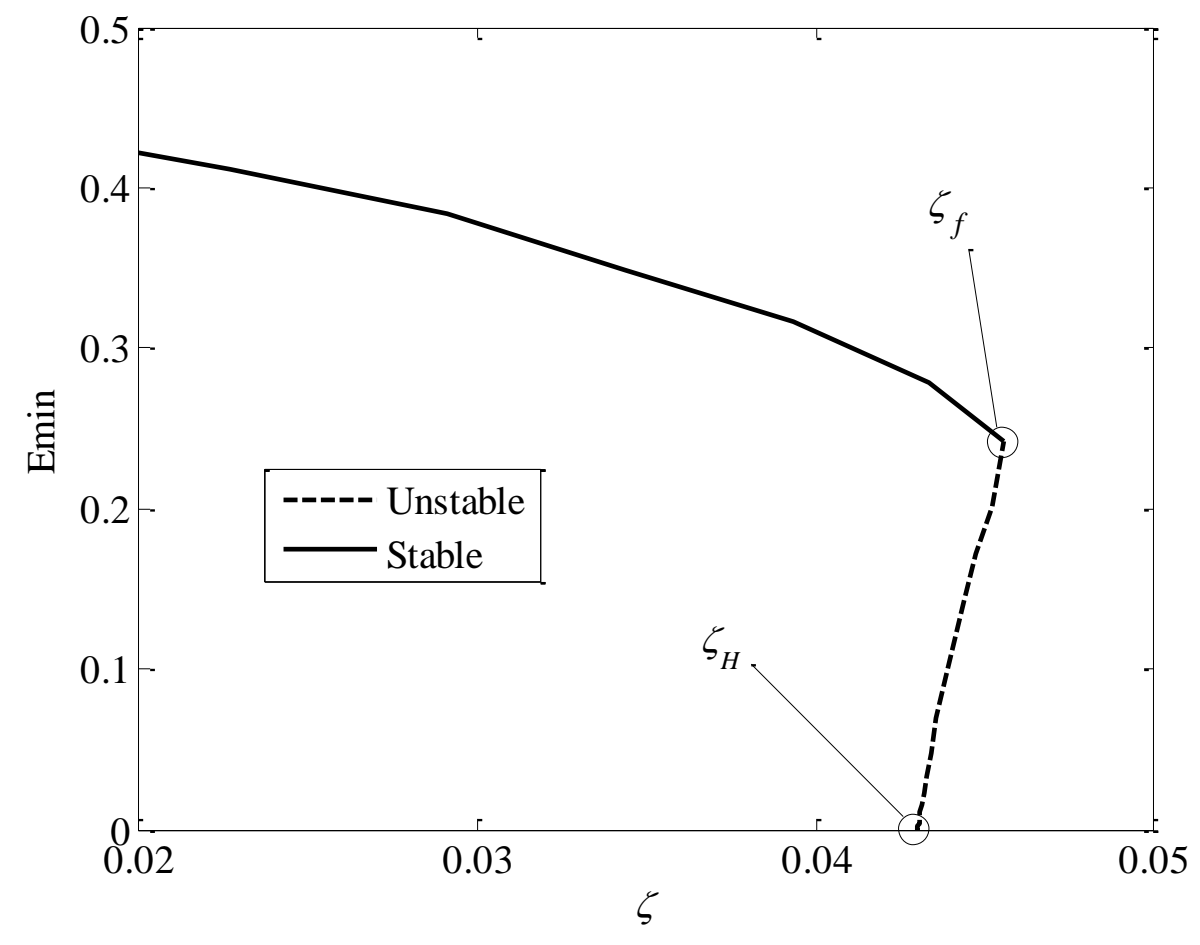

Fig. 8 Bifurcation diagrams as a function of $\zeta\left(\beta=0.874, x_{f}=0.3, \tau=0.02\right)$

To investigate the influence of damping on the dynamic behavior, the bifurcation diagram is displayed in Fig. 8. Since the damping effect could attenuate the oscillation, it is expected that the increased damping coefficient would stabilize the system, as provided in Fig. 8. For a large damping coefficient over $\zeta_{f}$, the system is stable to any finite magnitude perturbation. With the decrease of $\zeta$, the system exhibits bistability, wherein steady state solutions, small-amplitude unstable periodic solutions and largeamplitude stable limit cycles coexist. For a smaller damping coefficient over $\zeta_{H}$, the system is unstable even to infinitesimal perturbations. In the Rijke tube, the damping coefficient could be varied by changing the end conditions of the tube.

\subsection{Effect of bi-parameters}

A three dimensional bifurcation diagram was obtained by varying heat release coefficient $\beta$ and time delay $\tau$ simultaneously. Fig. 9 shows a good agreement with the 
bifurcation diagram reported in $[62,74]$. When the heat release coefficient $\beta$ is larger than the values of the Hopf points, an infinitesimal perturbation would result in a large-amplitude limit cycle, defined globally to be unstable. When $\beta$ is smaller than the values of the fold points, the steady state solution is stable for perturbations of any magnitude, appropriately defined to be globally stable. The region between the two lines indicates a bistable region for the system. In this region, the system will evolve to either steady state or stable limit cycle depending on the initial condition. Therefore, the Hopf bifurcation line denotes the linearly stability boundary and the fold bifurcation line indicates the nonlinearly stability boundary of the system.

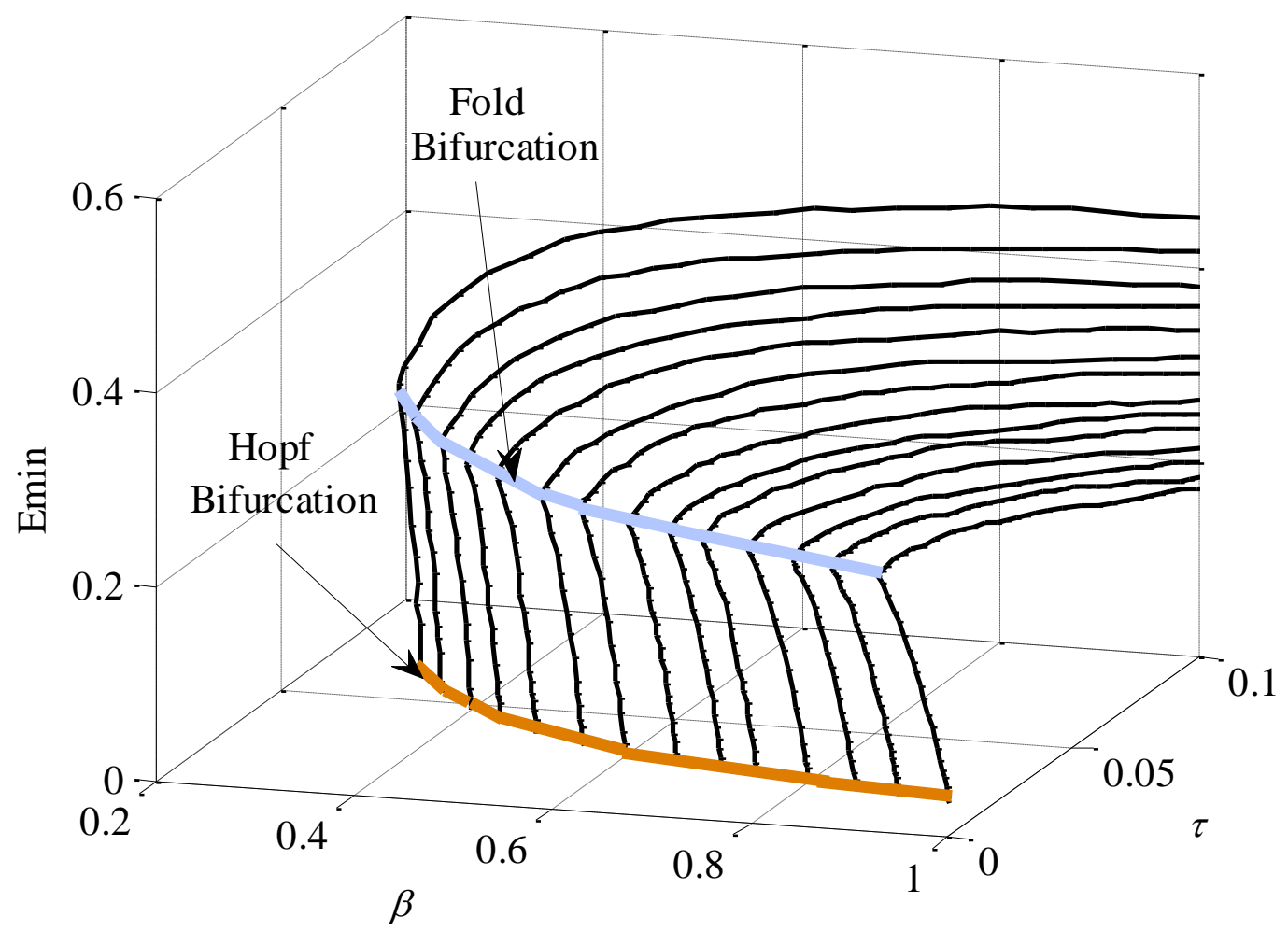

Fig. 9 Three dimensional bifurcation diagram for variation of $\beta$ and $\tau\left(\zeta=0.043, x_{f}=0.3\right)$

Figure 10 reveals a three-dimensional bifurcation diagram by varying damping coefficient $\zeta$ and time delay $\tau$ simultaneously, similar to the one obtained in [62]. The 
non/linear stability boundary as well as the bistable region could be identified obviously from Fig. 10.

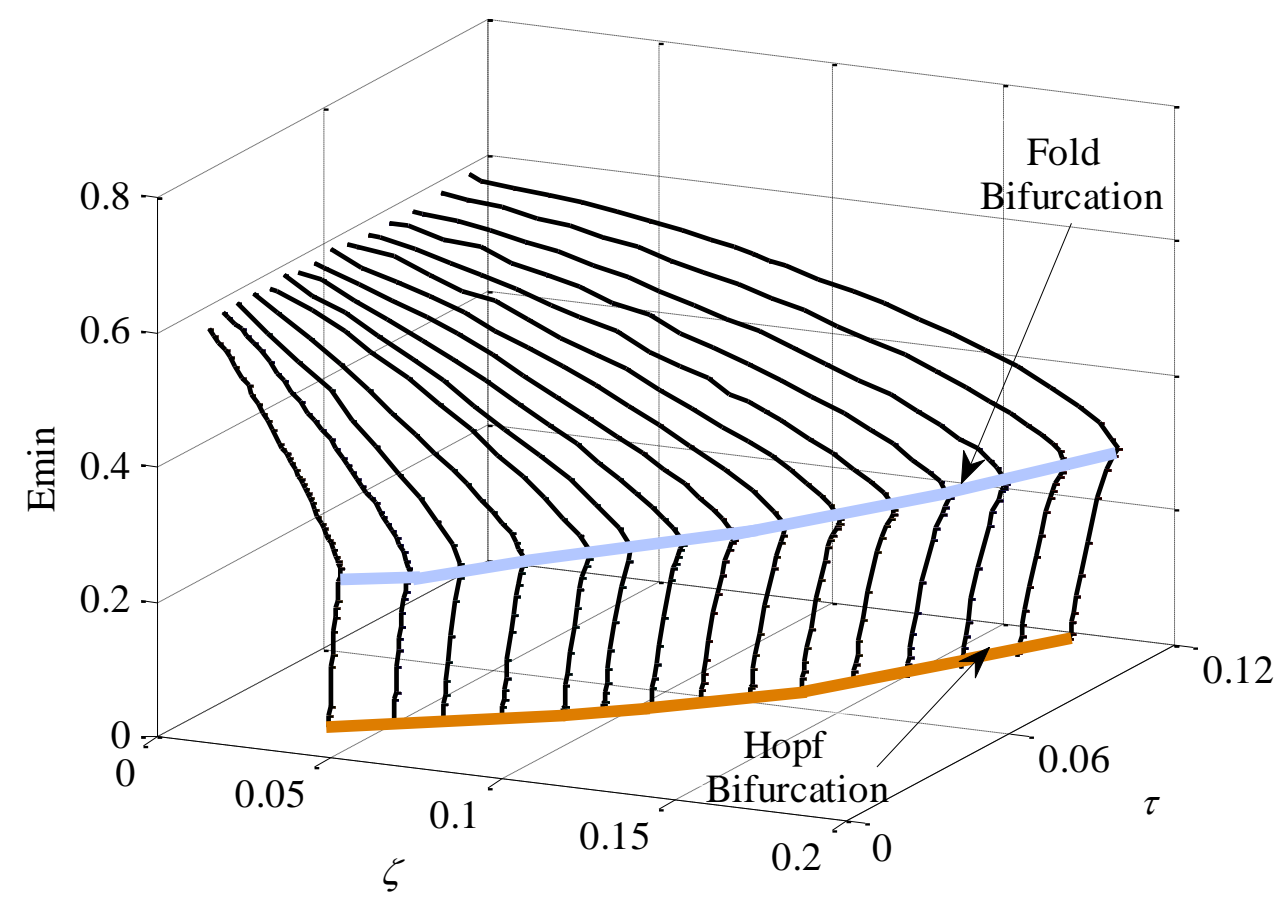

Fig. 10 Three dimensional bifurcation diagram for variation of $\zeta$ and $\tau\left(\beta=0.874, x_{f}=0.3\right)$

Two Hopf branches are continued by varying the heat release coefficient $\beta$, generic damping coefficient $\zeta$ with $\tau$ respectively, as shown in Fig. 11. In Fig. 11 (a), with fixed damping coefficient, a larger oscillating heat release would destabilize the system. Hence, the time delay needs to be smaller to make the system stable. In Fig. 11 (b), with fixed heat release, smaller damping coefficient will not balance the oscillation and smaller time delay contributes to the stability.

It is worth displaying, a two-dimensional projection of Fig. 9 in $\beta$ - $\tau$ plane as shown in Fig. 11(a) as well as the two-dimensional projection of Fig.10 in $\zeta-\tau$ plane in Fig. 11(b). 


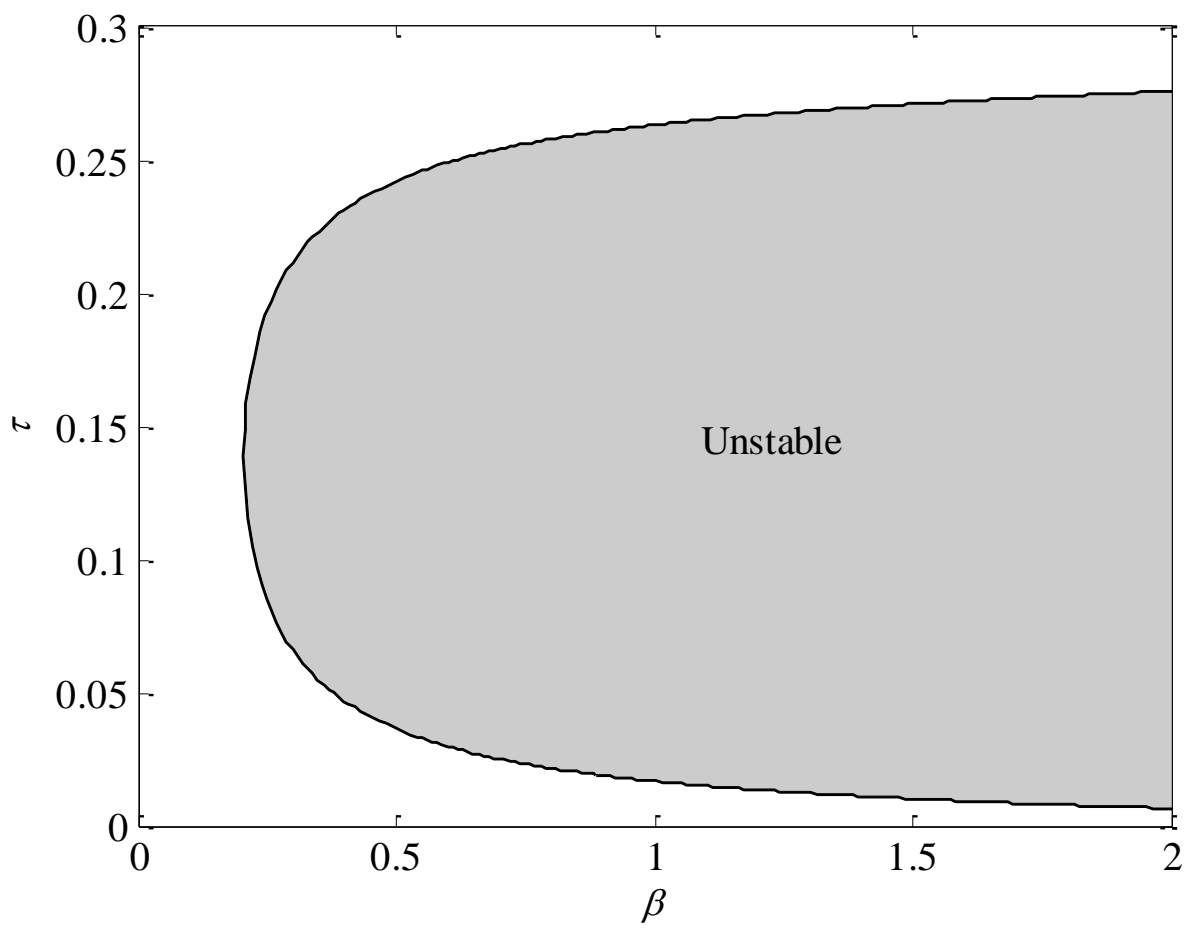

(a)

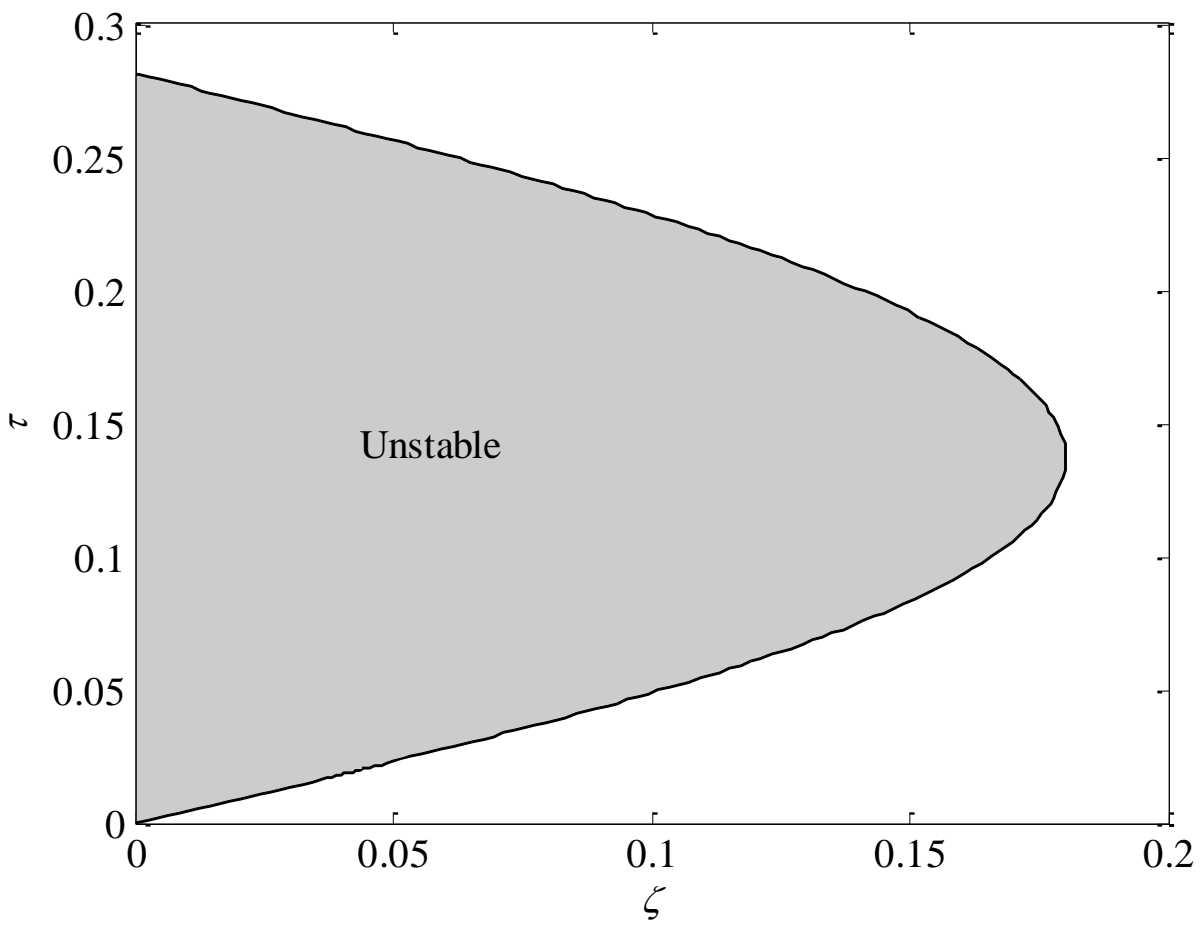

(b)

Fig. 11 Hopf branch of time delay $\tau$ with (a) $\beta\left(\zeta=0.043, x_{f}=0.3\right)$ and (b) $\zeta\left(\beta=0.043, x_{f}=0.3\right)$ 


\subsection{Comparison with Experimental Results}

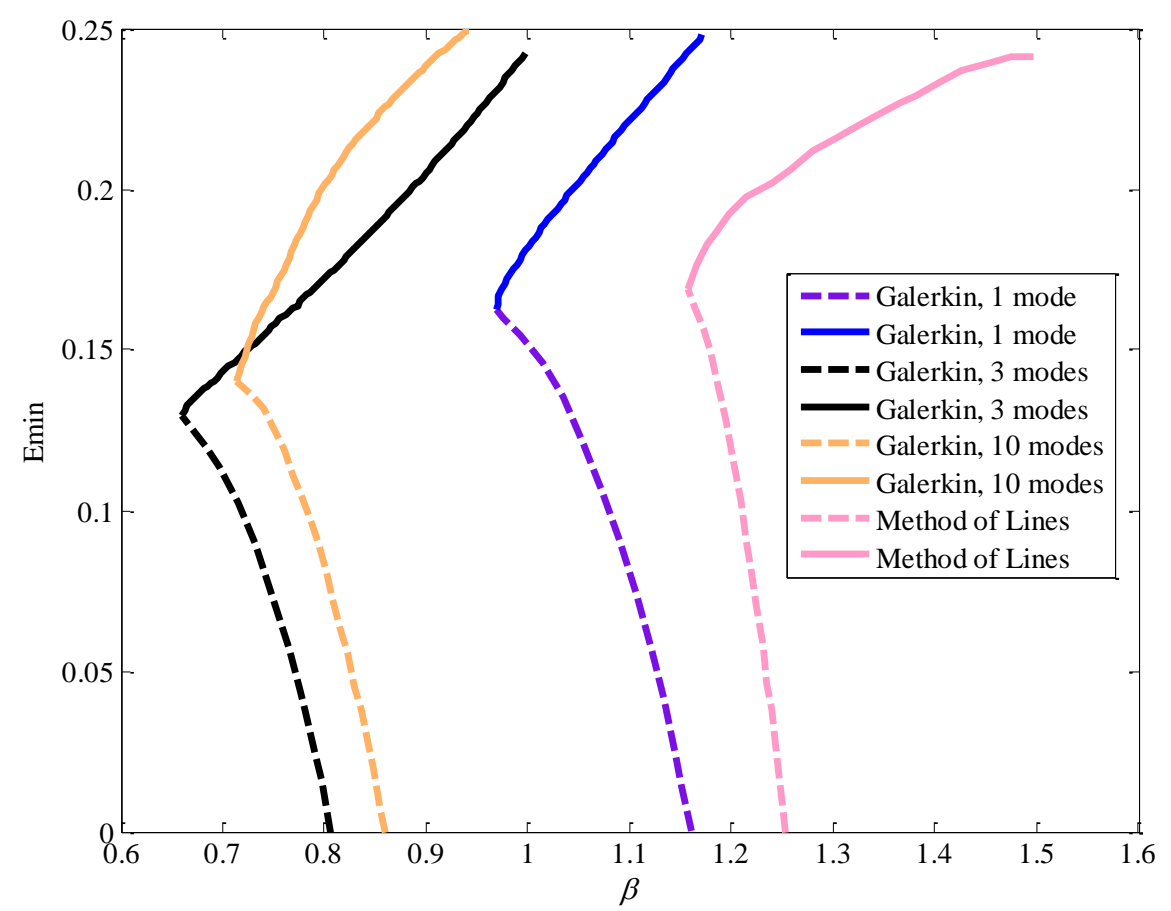

Fig. 12 Bifurcation diagram of the minimum acoustic energy as a function of heat release parameter $\beta$ $\left(\zeta=0.06, x_{f}=0.3, \tau=0.02\right)$ obtained by Method of Lines approach and Galerkin method using 1 mode, 3 modes and 10 mode respectively. Dashed line indicates the unstable periodic solution and solid line indicates the stable periodic solution.

Before comparing with the experimental results, a comparison of bifurcation diagram has been made between Galerkin method and MOL approach under equivalent condition. From the above figure, it can be seen that both Galerkin and MOL method have captured the subcritical Hopf bifurcation and fold bifurcation intrinsic in this dynamic system. The bifurcation diagrams are quite similar in terms of curve shape and tendency. However, the critical values $\beta_{H}$ and $\beta_{f}$ are predicted differently via these two methods. For Galerkin method, it is easily found that the results intensively depend on the number of modes chosen for the discretization of governing equations. With 1,3 and 10 mode used, the predicted $\beta_{H}$ equals to 1.161 , 0.806 and 0.859 respectively. Thus, one has to be very careful when choosing the 
mode number in order to get accurate results. In contrast, the discrepancy is much smaller using MOL with different discretization points selected along the axis direction, as shown in Fig. 5 in the grid independence check section. Additionally, the predicted critical values via MOL approach are larger than these predicted by Galerkin method. A larger $\beta_{H}$ indicates that the system is more difficult to lose stability with other bifurcation parameters set constant. Therefore, the system is more stable and the unstable area is smaller in the stability boundary diagram.

The stability boundary and nonlinear behavior inherent in the system have been studied experimentally by Matveev [58-59] and Song et al. [75]. In their experiments, the heat release coefficient $\beta$ and time delay $\tau$ were varied via changing the power supply for the hot wire gauze and mass flow rate simultaneously. Their relations could be determined from Eq. (8) and Eq. (19). Figure 13 shows a comparison of the stability boundary between the numerical results with the experimental and numerical data at different hot wire positions. When $x_{f}=0.25$, the predicted stability boundary displays good agreement with both the experimental results. When $x_{f}=0.625$, the stability boundary shows the same trend as the data obtained by Matveev [58].

It can be learnt from Fig. 11 that the areas encircled by the solid and dotted lines indicate the unstable areas and unsafe operation conditions in practice. As illustrated in Fig. 13, the unstable region is much smaller as predicted via MOL approach than Galerkin method, which indicates a more stable system and is consistent with the results as shown in Fig.12. In both cases, the predictions are more accurate than the results using Galerkin method and display good agreement with the results by experiments, which demonstrates the capability of this method in accurately capturing the nonlinear behaviors. 


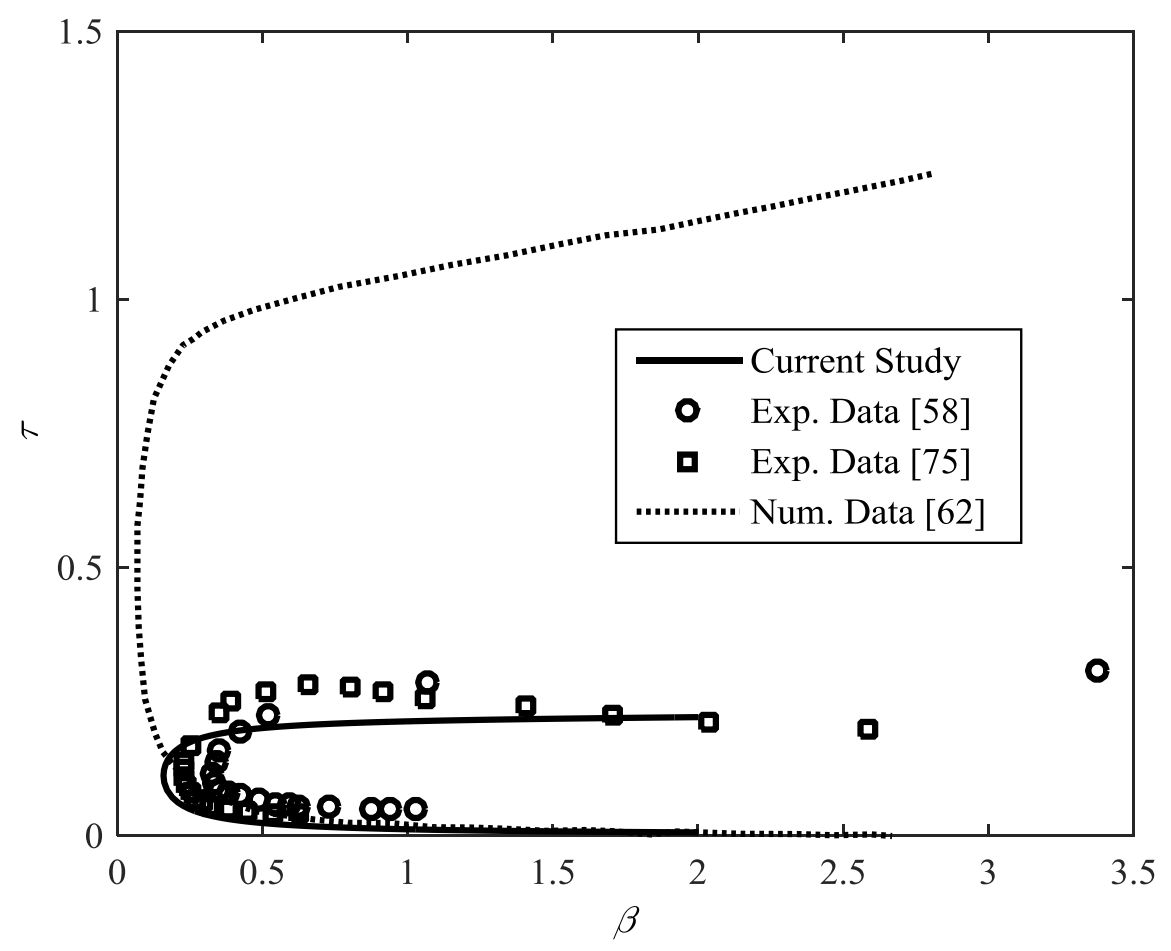

(a)

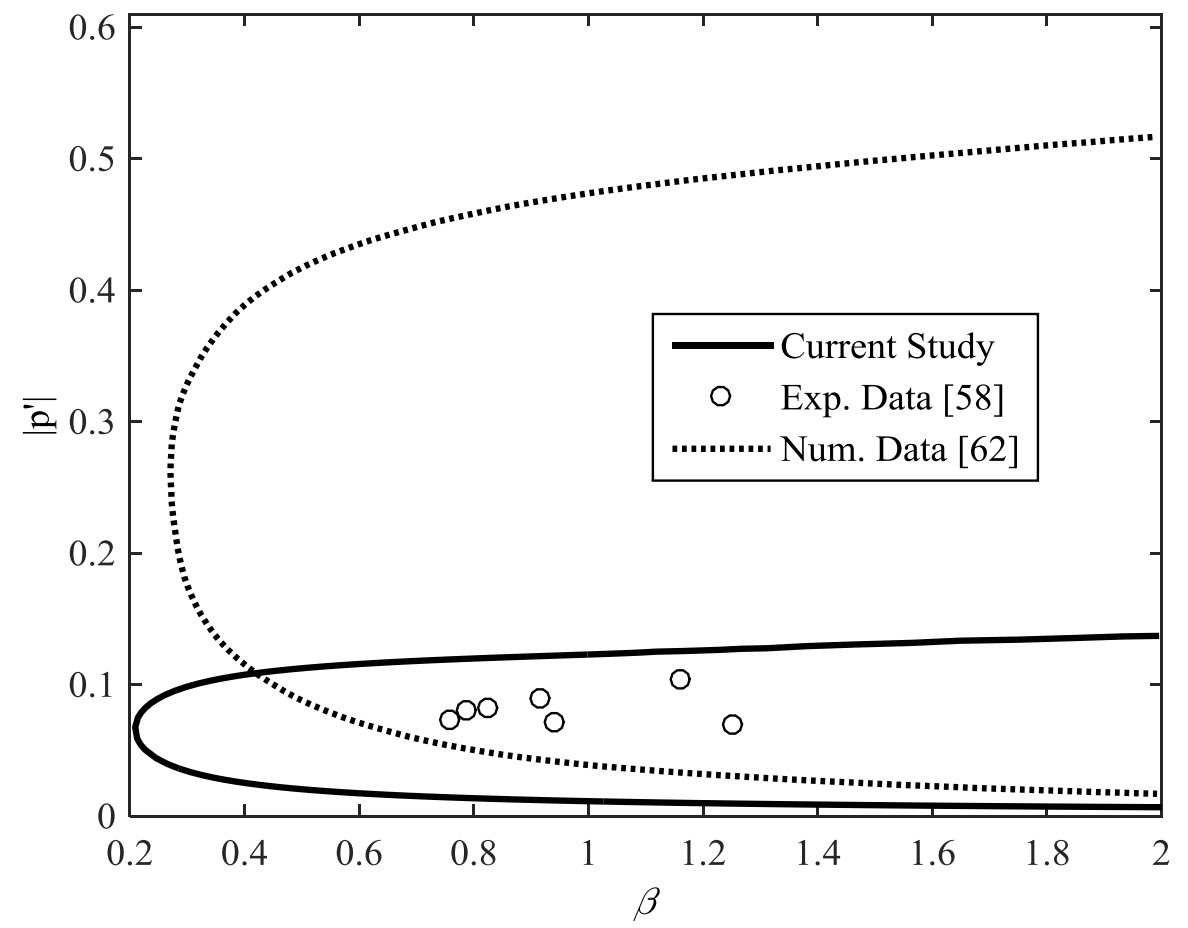

(b)

Fig. 13 Comparison between numerical results and experimental results of stability boundary

$$
\text { (a) } \zeta=0.0281, x_{f}=0.25 \text { (b) } \zeta=0.0281, x_{f}=0.625
$$

Besides, hysteresis was reported by Matveev [58] at the stability boundary for different mass flow rates. Fig. 14 exhibits the comparison of bifurcation behavior 
versus different time delays. The decrease of time delay would extend the linearly stable region and move the bifurcation curve forwards. To compare with the experimental data, the amplitude of the acoustic pressure was selected as a measure of the limit cycles. The numerical amplitude is close to the experimental data and the evolution tendency is similar between the numerical results and experimental data. The observed discrepancy regarding the results may be due to the relatively simple physical model adopted in current study. The configuration of the Rijke tube also has some influence vis-a-vis the experiments. However, the results have shown same order with the experimental data and more accurately captured nonlinear dynamics of the Rijke tube. The predictions obtained are encouraging and could reveal the rich nonlinear behaviors inherent in the Rijke tube thermoacoustic system. In a future study, it is hoped that the predictions will be more precise using more sophisticated models.

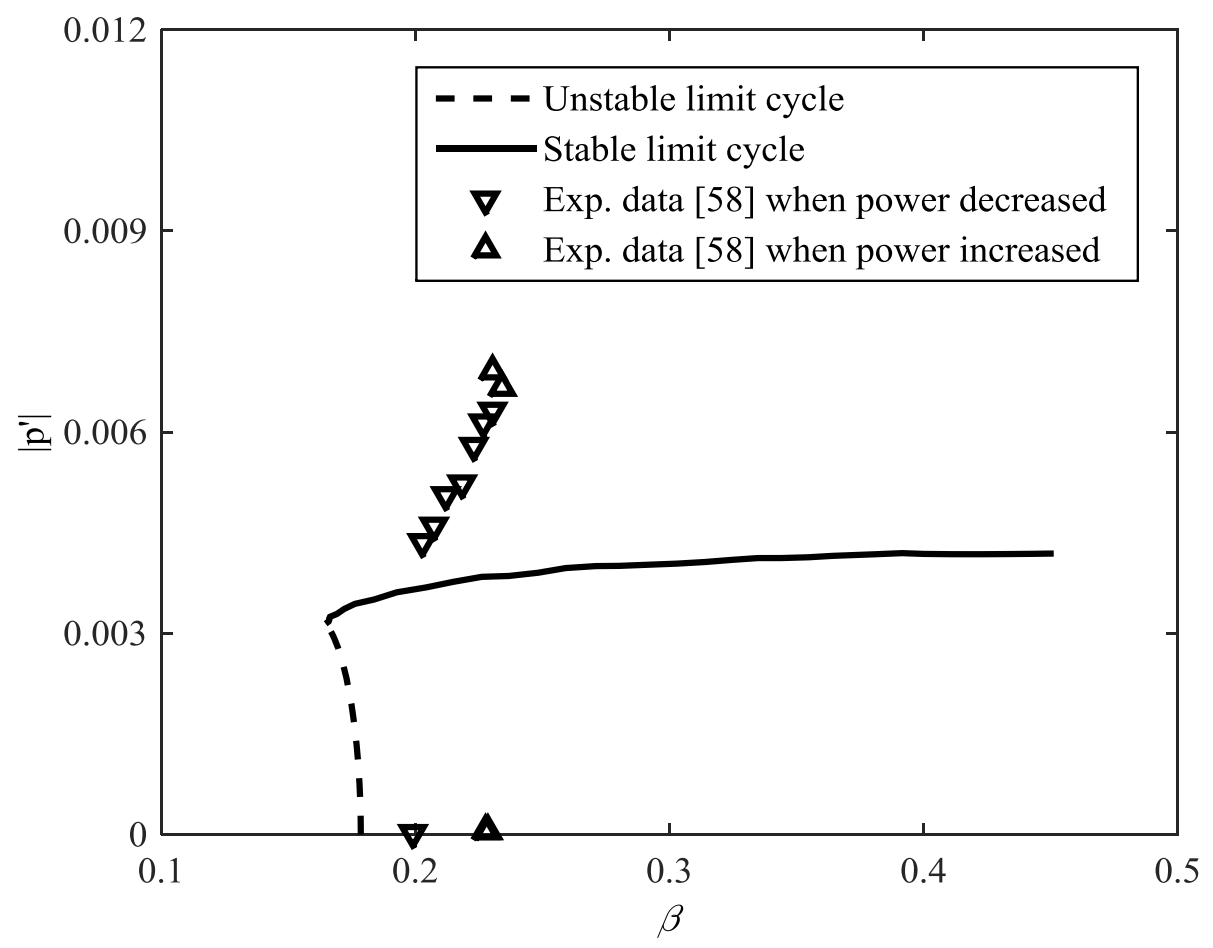

(a) $\tau=0.08$ 


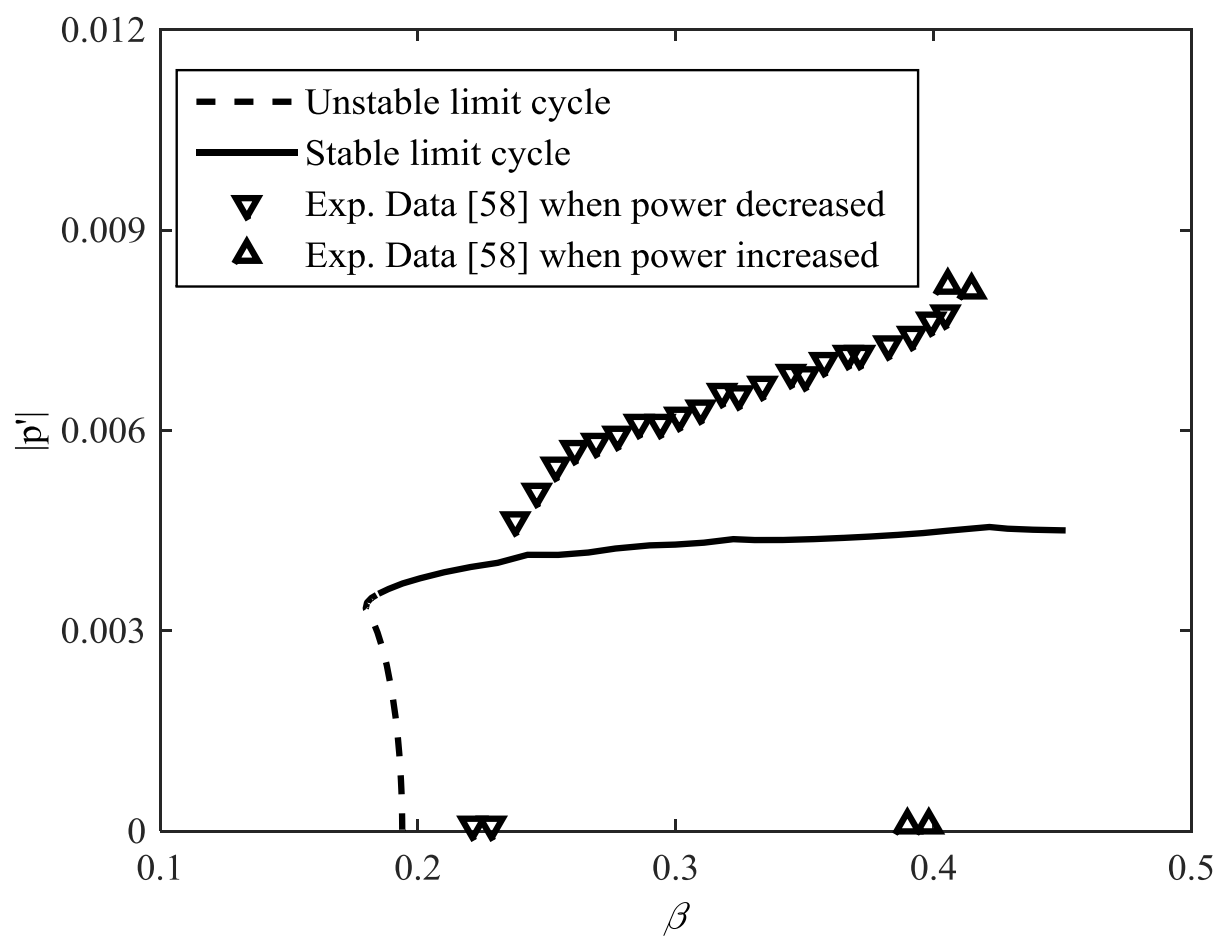

(b) $\tau=0.07$

Fig. 14 Comparison between numerical results and experimental results of bifurcation behavior

$$
\left(\zeta=0.0281, x_{f}=0.25\right)
$$

\section{Conclusions}

In this study, the dynamic behaviors of a horizontal Rijke tube thermoacoustic system are studied in detail. Method of Lines (MOL) technique is employed to discretize the governing equations and numerical continuation method is used to perform the bifurcation analysis.

The effects of heat release, damping and time delay are investigated by bifurcation diagrams for nonlinear analysis. Subcritical Hopf bifurcation and fold bifurcation are captured to reveal the nonlinear behavior of the system. The linear stability boundary, nonlinear stability boundary and bistable region are identified and limit cycles are predicted. It is demonstrated that the good predictions can be gained in comparison with the conventional method and, most importantly, the intrinsic nonlinear 
behaviours can be precisely captured compared with measurements as well as other predictions. Though the current heat release model employed in the paper is simplified, this method can be generalized for complex combustion and employed for the nonlinear stability of a generic time-delayed thermoacoustic combustion system.

\section{Acknowledgements}

The authors are indebted to Prof. Giovanni Samaey at KU Leuven for his valuable suggestions on the utilization of continuation method. X Yang acknowledges gratefully the support of President's Doctoral Scholar Award from the University of Manchester. S Lei would like to gratefully acknowledge the financial support by Industrial Development Agency (IDA) Ireland.

\section{Reference}

[1] Lei S, Turan A. Nonlinear/chaotic analysis, modelling and control of combustion instabilities due to vaporizing sprays. Chaos, Solitons \& Fractals 2009;42: 176679.

[2] Lieuwen TC, Yang V. Combustion Instabilities in Gas Turbine Engines: Operational Experience, Fundamental Mechanisms, and Modeling, AIAA (American Institute of Aeronautics \& Astronautics); 2005.

[3] Morgans A, Stow S. Model-based control of combustion instabilities in annular combustors. Combustion and Flame 2007;150: 380-99.

[4] Sondhauss C. Ueber die Schallschwingungen der Luft in erhitzten Glasröhren und in gedeckten Pfeifen von ungleicher Weite. Annalen der Physik 1850;155: 1-34.

[5] Rijke P. LXXI. Notice of a new method of causing a vibration of the air contained in a tube open at both ends. The London, Edinburgh, and Dublin Philosophical Magazine and Journal of Science 1859;17: 419-22. 
[6] Rayleigh JWS. The explanation of certain acoustical phenomena. Nature 1878;18: $319-21$.

[7] Culick F. Nonlinear behavior of acoustic waves in combustion chambers-I. Acta Astronautica 1976;3: 715-34.

[8] Culick FEC. Nonlinear behavior of acoustic waves in combustion chambers-II. Acta Astronautica 1976;3: 735-57.

[9] Dowling AP. The calculation of thermoacoustic oscillations. Journal of sound and vibration 1995;180: 557-81.

[10] Dowling AP. Nonlinear self-excited oscillations of a ducted flame. Journal of fluid mechanics 1997;346: 271-90.

[11] Dowling AP, Morgans AS. Feedback control of combustion oscillations. Annu Rev Fluid Mech 2005;37: 151-82.

[12] Dowling AP, Stow SR. Acoustic analysis of gas turbine combustors. Journal of propulsion and power 2003;19: 751-64.

[13] Han X, Li J, Morgans AS. Prediction of combustion instability limit cycle oscillations by combining flame describing function simulations with a thermoacoustic network model. Combustion and Flame 2015;162: 3632-47.

[14] Han X, Morgans AS. Simulation of the flame describing function of a turbulent premixed flame using an open-source LES solver. Combustion and Flame 2015;162: 1778-92.

[15] Juniper MP. Triggering in the horizontal Rijke tube: non-normality, transient growth and bypass transition. Journal of Fluid Mechanics 2011;667: 272-308.

[16] Heckl MA. Non-linear acoustic effects in the Rijke tube. Acta Acustica united with Acustica 1990;72: 63-71. 
[17] Hoeijmakers M, Arteaga IL, Kornilov V, Nijmeijer H, de Goey P. Accuracy assessment of thermoacoustic instability models using binary classification. International journal of spray and combustion dynamics 2013;5: 201-24.

[18] Hoeijmakers M, Kornilov V, Arteaga IL, de Goey P, Nijmeijer H. Intrinsic instability of flame-acoustic coupling. Combustion and Flame 2014;161: 2860-7.

[19] Hoeijmakers M, Kornilov V, Arteaga IL, de Goey P, Nijmeijer H. Flames in context of thermo-acoustic stability bounds. Proceedings of the Combustion Institute 2015;35: 1073-8.

[20] Kopitz J, Polifke W. CFD-based application of the Nyquist criterion to thermoacoustic instabilities. Journal of Computational Physics 2008;227: 6754-78.

[21] Mangesius H, Polifke W. A discrete-time, state-space approach for the investigation of non-normal effects in thermoacoustic systems. International journal of spray and combustion dynamics 2011;3: 331-50.

[22] C. Silva TR, W. Polifke, and L. Magri. Uncertainty quantification of growth rates of thermoacoustic instability by an adjoint Helmholtz solver. Proceedings of ASME 2016 Turbo Expo: Turbomachinery Technical Conference \& Exposition, GT2016-57659, Seoul, Korea 2016.

[23] Selimefendigil F, Polifke W. A frequency domain system model with coupled modes for limit cycle prediction of thermoacoustic systems. Int J Spray Combust Dynam 2011;3: 303-30.

[24] Hermeth S, Staffelbach G, Gicquel LY, Anisimov V, Cirigliano C, Poinsot T. Bistable swirled flames and influence on flame transfer functions. Combustion and Flame 2014;161: 184-96. 
[25] Martin CE, Benoit LJ-L, Sommerer Y, Nicoud F, Poinsot T. Large-eddy simulation and acoustic analysis of a swirled staged turbulent combustor. AIAA journal 2006;44: 741-50.

[26] Hermeth S, Staffelbach G, Gicquel LY, Poinsot T. LES evaluation of the effects of equivalence ratio fluctuations on the dynamic flame response in a real gas turbine combustion chamber. Proceedings of the Combustion Institute 2013;34: 3165-73.

[27] Schmitt P, POINSOT T, Schuermans B, Geigle K. Large-eddy simulation and experimental study of heat transfer, nitric oxide emissions and combustion instability in a swirled turbulent high-pressure burner. Journal of fluid mechanics 2007;570: 17-46.

[28] Boudier G, Lamarque N, Staffelbach G, Gicquel L, Poinsot T. Thermo-acoustic stability of a helicopter gas turbine combustor using large eddy simulation. International journal of Aeroacoustics 2009;8: 69-93.

[29] Nicoud F, Benoit L, Sensiau C, Poinsot T. Acoustic modes in combustors with complex impedances and multidimensional active flames. AIAA journal 2007;45: $426-41$.

[30] Boudy F, Durox D, Schuller T, Candel S. Analysis of limit cycles sustained by two modes in the flame describing function framework. Comptes rendus mecanique 2013;341: 181-90.

[31] Candel S, Durox D, Schuller T, Palies P, Bourgouin J-F, Moeck JP. Progress and challenges in swirling flame dynamics. Comptes rendus mecanique 2012;340: 758-68. 
[32] Moeck JP, Bourgouin J-F, Durox D, Schuller T, Candel S. Nonlinear interaction between a precessing vortex core and acoustic oscillations in a turbulent swirling flame. Combustion and Flame 2012;159: 2650-68.

[33] Noiray N, Durox D, Schuller T, Candel S. A unified framework for nonlinear combustion instability analysis based on the flame describing function. Journal of fluid mechanics 2008;615: 139-67.

[34] Ducruix Sé, Schuller T, Durox D, Candel b. Combustion dynamics and instabilities: Elementary coupling and driving mechanisms. Journal of propulsion and power 2003;19: 722-34.

[35] Balasubramanian K, Sujith R. Thermoacoustic instability in a Rijke tube: Nonnormality and nonlinearity. Physics of Fluids (1994-present) 2008;20: 044103.

[36] Balasubramanian K, Sujith R. Non-normality and nonlinearity in combustionacoustic interaction in diffusion flames. Journal of fluid mechanics 2008;594: 2957.

[37] Kabiraj L, Saurabh A, Wahi P, Sujith R. Route to chaos for combustion instability in ducted laminar premixed flames. Chaos: An Interdisciplinary Journal of Nonlinear Science 2012;22: 023129.

[38] Kabiraj L, Sujith R. Nonlinear self-excited thermoacoustic oscillations: intermittency and flame blowout. Journal of fluid mechanics 2012;713: 376-97.

[39] Nair V, Thampi G, Sujith R. Intermittency route to thermoacoustic instability in turbulent combustors. Journal of fluid mechanics 2014;756: 470-87.

[40] You D, Huang Y, Yang V. A generalized model of acoustic response of turbulent premixed flame and its application to gas-turbine combustion instability analysis. Combustion Science and Technology 2005;177: 1109-50.

[41] Yang V. Liquid rocket engine combustion instability, AIAA; 1995. 
[42] Yang V. Modeling of supercritical vaporization, mixing, and combustion processes in liquid-fueled propulsion systems. Proceedings of the Combustion Institute 2000;28: 925-42.

[43] Campa G, Camporeale SM. Prediction of the thermoacoustic combustion instabilities in practical annular combustors. Journal of Engineering for Gas Turbines and Power 2014;136: 091504.

[44]Campa G, Camporeale SM, Cosatto E, Mori G. Thermoacoustic analysis of combustion instability through a distributed flame response function. ASME Turbo Expo 2012: Turbine Technical Conference and Exposition. American Society of Mechanical Engineers2012. pp. 179-88.

[45]Camporeale S, Fortunato B, Campa G. A finite element method for threedimensional analysis of thermo-acoustic combustion instability. Journal of Engineering for Gas Turbines and Power 2011;133: 011506.

[46]Culick F. A review of calculations for unsteady burning of a solid propellant. AIAA journal 1968;6: 2241-55.

[47]Feldman K. Review of the literature on Rijke thermoacoustic phenomena. Journal of sound and vibration 1968;7: 83-9.

[48]Lieuwen T. Modeling premixed combustion-acoustic wave interactions: A review. Journal of propulsion and power 2003;19: 765-81.

[49] Putnam A. Combustion-Driven Oscillations in Industry,(1971). Elesevier Pub.

[50] Dupere ID, Dowling AP. The use of Helmholtz resonators in a practical combustor. Journal of Engineering for Gas Turbines and Power 2005;127: 26875.

[51] Zhao D, Morgans AS. Tuned passive control of combustion instabilities using multiple Helmholtz resonators. Journal of sound and vibration 2009;320: 744-57. 
[52] Zhao D, Morgans AS, Dowling AP. Tuned passive control of acoustic damping of perforated liners. AIAA journal 2011;49: 725-34.

[53] Altay HM, Hudgins DE, Speth RL, Annaswamy AM, Ghoniem AF. Mitigation of thermoacoustic instability utilizing steady air injection near the flame anchoring zone. Combustion and Flame 2010;157: 686-700.

[54] Hathout J, Fleifil M, Annaswamy A, Ghoniem A. Combustion instability active control using periodic fuel injection. Journal of propulsion and power 2002;18: $390-9$.

[55] Raun R, Beckstead M, Finlinson J, Brooks K. A review of Rijke tubes, Rijke burners and related devices. Progress in Energy and Combustion Science 1993;19: 313-64.

[56] Zhao D. Transient growth of flow disturbances in triggering a Rijke tube combustion instability. Combustion and Flame 2012;159: 2126-37.

[57] Hantschk CC, Vortmeyer D. Numerical simulation of self-excited thermoacoustic instabilities in a Rijke tube. Journal of Sound and Vibration 1999;227: 511-22.

[58] Matveev K. Thermoacoustic instabilities in the Rijke tube: experiments and modeling: California Institute of Technology; 2003.

[59] Matveev K, Culick F. A study of the transition to instability in a Rijke tube with axial temperature gradient. Journal of Sound and Vibration 2003;264: 689-706.

[60] Ananthkrishnan N, Deo S, Culick FE. Reduced-order modeling and dynamics of nonlinear acoustic waves in a combustion chamber. Combustion Science and Technology 2005;177: 221-48.

[61] Heckl MA, Howe M. Stability analysis of the Rijke tube with a Green's function approach. Journal of Sound and Vibration 2007;305: 672-88. 
[62] Subramanian P, Mariappan S, Sujith R, Wahi P. Bifurcation analysis of thermoacoustic instability in a horizontal Rijke tube. International journal of spray and combustion dynamics 2010;2: 325-55.

[63] Noble AC, King GB, Laurendeau NM, Gord JR, Roy S. Nonlinear Thermoacoustic Instability Dynamics in a Rijke Tube. Combustion Science and Technology 2012;184: 293-322.

[64] Mikhlin SG. Variational methods in mathematical physics, Pergamon Press; [distributed by Macmillan, New York]; 1964.

[65] Schiesser WE. The Numerical Method of Lines: Integration of Partial Differential Equations, Academic Press; 1991.

[66] Engelborghs K, Luzyanina T, Roose D. Numerical bifurcation analysis of delay differential equations using DDE-BIFTOOL. ACM Transactions on Mathematical Software (TOMS) 2002;28: 1-21.

[67] Engelborghs K, Luzyanina T, Samaey G. DDE-BIFTOOL: a Matlab package for bifurcation analysis of delay differential equations. Department of Computer Science, Katholieke Universiteit Leuven, Leuven, Belgium 2000.

[68] Rayleigh L. The Explanation of Certain Acoustical Phenomena. Nature 1878;18: $319-21$.

[69] Howe MS. Acoustics of fluid-structure interactions, Cambridge University Press; 1998.

[70] Doedel E, Keller HB, Kernevez JP. Numerical analysis and control of bifurcation problems (I): Bifurcation in finite dimensions. International journal of bifurcation and chaos 1991;1: 493-520.

[71] Seydel R. Practical bifurcation and stability analysis, Springer; 2010. 
[72] Waugh I. Methods for analysis of nonlinear thermoacoustic systems: University of Cambridge; 2013.

[73] Sarpotdar SM, Ananthkrishnan N, Sharma S. The Rijke Tube-A Thermoacoustic Device. RESONANCE 2003.

[74] Lighthill MJ. The response of laminar skin friction and heat transfer to fluctuations in the stream velocity. Proceedings of the Royal Society of London Series A Mathematical and Physical Sciences 1954;224: 1-23.

[75] Song W-S, Lee S, Shin D-S, Na Y. Thermo-acoustic instability in the horizontal Rijke tube. Journal of mechanical science and technology 2006;20: 905-13. 